\title{
The vertebrate ancestral repertoire of visual opsins, transducin alpha subunits and oxytocin/ vasopressin receptors was established by duplication of their shared genomic region in the two rounds of early vertebrate genome duplications
}

David Lagman ${ }^{1 \dagger}$, Daniel Ocampo Daza ${ }^{1 \dagger}$, Jenny Widmark ${ }^{1}$, Xesús M Abalo ${ }^{1}$, Görel Sundström ${ }^{1,2}$ and Dan Larhammar ${ }^{1 *}$

\begin{abstract}
Background: Vertebrate color vision is dependent on four major color opsin subtypes: RH2 (green opsin), SWS1 (ultraviolet opsin), SWS2 (blue opsin), and LWS (red opsin). Together with the dim-light receptor rhodopsin (RH1), these form the family of vertebrate visual opsins. Vertebrate genomes contain many multi-membered gene families that can largely be explained by the two rounds of whole genome duplication (WGD) in the vertebrate ancestor (2R) followed by a third round in the teleost ancestor (3R). Related chromosome regions resulting from WGD or block duplications are said to form a paralogon. We describe here a paralogon containing the genes for visual opsins, the G-protein alpha subunit families for transducin (GNAT) and adenylyl cyclase inhibition (GNAI), the oxytocin and vasopressin receptors (OTNP-R), and the L-type voltage-gated calcium channels (CACNA1-L).

Results: Sequence-based phylogenies and analyses of conserved synteny show that the above-mentioned gene families, and many neighboring gene families, expanded in the early vertebrate WGDs. This allows us to deduce the following evolutionary scenario: The vertebrate ancestor had a chromosome containing the genes for two visual opsins, one GNAT, one GNAl, two OTNP-Rs and one CACNA1-L gene. This chromosome was quadrupled in 2R. Subsequent gene losses resulted in a set of five visual opsin genes, three GNAT and GNAI genes, six OTNP-R genes and four CACNA1-L genes. These regions were duplicated again in 3R resulting in additional teleost genes for some of the families. Major chromosomal rearrangements have taken place in the teleost genomes. By comparison with the corresponding chromosomal regions in the spotted gar, which diverged prior to $3 R$, we could time these rearrangements to post-3R.

(Continued on next page)
\end{abstract}

\footnotetext{
*Correspondence: Dan.Larhammar@neuro.uu.se

${ }^{\dagger}$ Equal contributors

'Department of Neuroscience, Science for Life Laboratory, Uppsala University,

Box 593, SE-75124 Uppsala, Sweden

Full list of author information is available at the end of the article
} 
(Continued from previous page)

Conclusions: We present an extensive analysis of the paralogon housing the visual opsin, GNAT and GNAI, OTNP-R, and CACNA1-L gene families. The combined data imply that the early vertebrate WGD events contributed to the evolution of vision and the other neuronal and neuroendocrine functions exerted by the proteins encoded by these gene families. In pouched lamprey all five visual opsin genes have previously been identified, suggesting that lampreys diverged from the jawed vertebrates after $2 \mathrm{R}$.

Keywords: Visual opsins, Whole genome duplications, Chromosome rearrangements, Opsin evolution, Oxytocin receptors, Vasopressin receptors, G-protein alpha transducing subunits, Voltage-gated calcium channels

\section{Background}

Color vision is the ability to distinguish light of different wavelengths. This property has arisen independently in vertebrates and invertebrates through duplications of the genes encoding ciliary opsins and rhabdomeric opsins, respectively [1]. In the vertebrates, three types of cones displaying distinct wavelength properties were initially described by Ragnar Granit who received the Nobel Prize for these achievements in 1967. Later studies, including molecular cloning of the visual opsins in various vertebrates, have shown that four major color opsin subtypes existed in early vertebrates [2-4]. Thus, together with the dim-light (scotopic) receptor rhodopsin expressed in rods, the family of visual opsins encompassed five members in early vertebrates: $R H 1$ (rhodopsin), RH2 (green opsin), SWS1 (ultraviolet opsin), SWS2 (blue opsin), and $L W S$ (red opsin).

Subsequently, the gene repertoire has changed by gains and losses of opsin genes in the different vertebrate lineages. For instance, the ancestor of placental mammals lost RH2 and SWS2 and extant mammals use SWS1 for vision in the blue part of the spectrum. Primates have a duplicate of LWS that has mutated to have its absorption maximum in green, namely OPN1MW. The platypus has retained SWS2 but lost SWS1. Other losses have occurred in frogs (RH2), in the coelacanth Latimeria chalumnae, and in cartilaginous fishes. In teleost fishes, on the other hand, many additional duplicates have arisen [5].

Phylogenetic analyses of the five visual opsin sequences in chicken showed that the rhodopsin sequence $R H 1$ was most closely related to the green opsin $R H 2$, suggesting that this gene duplication was the most recent and that the four color opsins existed before rhodopsin [6]. Thus, it appeared that color vision was ancestral to the dim-light vision and the appearance of rhodopsin and rod photoreceptors facilitated the adaptation to nocturnal environments. As a rhodopsin sequence had already been described in a lamprey, this implied that all five opsins existed before the divergence of lampreys and gnathostomes, i.e., jawed vertebrates. Subsequently, also the four color opsins have been described in pouched lamprey (Geotria australis) [7].
Although the visual opsin gene duplications must have taken place before the lamprey-gnathostome divergence, it has remained unclear how they happened. We and others have previously reported that numerous gene families expanded in the two genome doublings, i.e. tetraploidizations, that took place before the radiation of gnathostomes [8-11] usually called $2 \mathrm{R}$ for two rounds of genome doubling. The two tetraploidizations resulted in a large number of quartets of related chromosome regions, and each such quartet is called a paralogon. Subsequently, a third tetraploidization, 3R, took place in the lineage leading to teleost fishes [12]. By investigating gene families sharing chromosome regions, we have been able to deduce the evolution of multiple neuronal and endocrine gene families as well as their neighbors, namely the opioid peptides [13] and receptors [14], neuropeptide Y-family peptides [15] and receptors [16,17], voltage-gated sodium channels and their neighboring TGF- $\beta$ receptors [18], the IGFBP family [19], the paralemmin family [20] and, more recently, the three transducin subunit gene families activated by visual opsins [21]. All of these families received additional members in $2 \mathrm{R}$ and all but one expanded further in 3R. Chromosomal positions thereby constitute a useful additional type of information for analyses of gene families, especially families that display different evolutionary rates among members or over time, both of which seem to afflict the visual opsins.

We have previously performed sequence-based phylogenetic analyses of several gene families in the phototransduction cascade, and also investigated their chromosomal positions in the human genome [21-23]. These analyses suggested that most of the phototransduction gene families expanded in the basal vertebrate tetraploidizations, including the visual opsin family. In our first study, we proposed that RH1, SWS1 and LWS arose as a result of duplications of a large chromosome block [23]. Because teleost fishes and birds have the LWS and SWS2 genes in close proximity on the same chromosome [24-26], we suggested a scenario where two adjacent visual opsin genes were quadrupled by chromosome duplications [22]. However, the paralogon harboring the opsin genes seemed to have undergone major rearrangements and only a few adjacent gene families were identified, thereby making our 
conclusions uncertain. Interestingly, one of the neighboring gene families was the transducin alpha subunit family (GNAT), involved in the phototransduction cascade, as well as its adjacent relative $\mathrm{G}$ protein alpha inhibiting subunit (GNAI) gene [21-23]. The GNAT family includes three genes located on three of the visual opsin chromosomes in several vertebrates, each flanked by a more distantly related GNAI gene $[21,27,28]$.

In parallel, independent analyses in our laboratory of the oxytocin/vasopressin receptor (OT/VP-R) genes and the L-type voltage-gated calcium channel alpha subunit (CACNA1-L) genes converged to reveal large chromosomal regions that share evolutionary history with the visual opsin, GNAT and GNAI genes. The pituitary peptide hormones oxytocin and vasopressin have previously been reported to have five to six ancestral vertebrate receptors (OT/VP-R) based on phylogenetic analyses $[29,30]$ : one oxytocin receptor, OTR, encoded by OXTR genes, and four to five vasopressin receptors, including V1A $(A V P R 1 A), \mathrm{V} 1 \mathrm{~B}(A V P R 1 B)$ as well as several types of $\mathrm{V} 2$ receptors. We report here that the OT/VP-R family genes are located in the proposed visual opsin paralogon, thus resolving the issue of the evolutionary relationships between the ancestral members. Finally, the L-type voltage-gated calcium channel alpha subunits (CACNA1-L) form a family with four members whose genes are located on the visual opsin chromosomes.

We have used these gene families as starting points for extensive analyses of conserved synteny in species representing several vertebrate classes. We report here that these five main gene families and 34 neighboring gene families comprise large paralogous chromosomal regions with extensive similarities to one another that can most parsimoniously be explained by quadruplication of a large ancestral chromosome region. These results define the time points for expansion of the visual opsin family as well as the transducin alpha family, the oxytocin/vasopressin receptors, and the L-type voltage-gated calcium channels. Furthermore, our results have implications for the divergence time point of lampreys and jawed vertebrates relative to the two basal vertebrate tetraploidizations.

\section{Results}

We used amino acid sequences identified in genome databases to produce alignments and phylogenetic trees for the visual opsins, the oxytocin and vasopressin receptors (OT/VP-R), the G-protein alpha transducing (GNAT) and inhibiting (GNAI) subunits, as well as the L-type voltage-gated calcium channel alpha subunits (CACNA1-L). Detailed information on these gene families, including database identifiers, location data, genome assembly information and annotation notes for all identified sequences, is provided in Additional file 1. Topologies of the visual opsin and OT/VP-R gene family trees are presented in this section and we have recently published our phylogenetic analyses of the GNAT and GNAI gene families [21]. The phylogenetic analyses of the CACNA1-L family, which have not been published previously, are included in Additional file 2: Figures S7 and S8. These gene families have members located in overlapping regions of the human genome putatively considered as one paralogon and are henceforth referred to as the "main" gene families of this study.

We have also made phylogenetic trees for 34 neighboring gene families identified in the chromosomal regions of the main gene families. The positional data from the main gene families and the neighboring gene families have been compared between the genomes of human, chicken, zebrafish, three-spined stickleback and spotted gar. This combination of phylogenetic and synteny data is the basis for the description of our results below.

\section{Phylogenetic analyses of vertebrate visual opsin genes}

Genes for the five ancestral types of visual opsins present in the vertebrate ancestor (LWS, SWS1, SWS2, RH2 and RH1) [4] were identified in sarcopterygians (including tetrapods and coelacanth) and actinopterygians (ray-finned fishes, including teleosts and spotted gar). These results are summarized in Table 1 . In the phylogenetic analyses these sequences form five well-supported clusters: The LWS cluster forms a basal separate branch while the SWS1, SWS2, RH2 and RH1 cluster together with high support (Figure 1). This topology is supported by neighbor joining (NJ) and phylogenetic maximum likelihood (PhyML) methods (Additional file 2: Figures S1 and S2). The trees were rooted with the human OPN3 sequence, as the OPN3 gene has been shown to diverge before the diversification of the visual opsin genes [31,32]. Pinopsins and the vertebrate ancient (V/A) opsins often cluster with the visual opsins in phylogenetic analyses [5,31-33], and there are also related opsins in the tunicate Ciona intestinalis called Ci-opsin 1 and Ci-opsin2 [34]. The overall topology presented in Figure 1 is not affected by the inclusion of these sequences in additional phylogenetic analyses (Additional file 2: Figures S3 and S4). All opsin phylogenetic tree files and the alignments they were made from are provided as a citable file set with a stable identifier - see reference [35].

\section{Visual opsin gene repertoires}

The repertoires of visual opsins in mammals and birds have been described in detail in numerous previous studies, and the anole lizard (Anolis carolinensis) opsin genes have been described more recently [37]. These data are consistent with our analyses (Figure 1), which confirm that the ancestral repertoire in jawed vertebrates 
Table 1 Summary of visual opsin sequences analyzed in this study

\begin{tabular}{|c|c|c|c|c|c|}
\hline & $\begin{array}{l}\text { Species (genome } \\
\text { assembly version) }\end{array}$ & $\begin{array}{l}\text { Sequence names in } \\
\text { phylogenetic analyses a }\end{array}$ & Type $^{b}$ & $\begin{array}{l}\text { Chromosome, linkage group, } \\
\text { or genomic scaffold }\end{array}$ & Loc. (Mb) \\
\hline \multicolumn{6}{|l|}{ Sarcopterygians } \\
\hline \multirow[t]{11}{*}{ Mammals } & Human & Hsa X OPN1LW & LWS & $x$ & 153.41 \\
\hline & (GRCh37) & Hsa X OPN1MW2 & LWS & $x$ & 153.49 \\
\hline & & Hsa X OPN1MW & LWS & $x$ & 153.45 \\
\hline & & Hsa 3 RHO & $\mathrm{RH} 1$ & 3 & 129.25 \\
\hline & & Hsa 7 OPN1SW & SWS1 & 7 & 128.41 \\
\hline & Mouse & Mmu X Opn1mw & LWS & $x$ & 71.37 \\
\hline & (NCBIM37) & Mmu 6 Rho & $\mathrm{RH} 1$ & 6 & 115.88 \\
\hline & & Mmu 6 Opn1sw & SWS1 & 6 & 29.33 \\
\hline & Grey short-tailed opossum & Mdo X LWS & LWS & $x$ & 14.66 \\
\hline & (MonDom5) & Mdo 6 RH1 & $\mathrm{RH} 1$ & 6 & 246.61 \\
\hline & & Mdo 8 SWS1 & SWS1 & 8 & 188.61 \\
\hline \multirow[t]{5}{*}{ Birds } & Chicken & Gga LWS & LWS & c & \\
\hline & (WASHUC2) & Gga 12 RH1 & $\mathrm{RH} 1$ & 12 & 20.16 \\
\hline & & Gga 26 RH2 & $\mathrm{RH} 2$ & 26 & 4.38 \\
\hline & & Gga SWS1 & SWS1 & c & \\
\hline & & Gga SWS2 & SWS2 & c & \\
\hline \multirow[t]{5}{*}{ Non-avian reptiles } & Carolina anole lizard & Aca 2 LWS & LWS & 2 & 88.66 \\
\hline & (AnoCar2.0) & Aca $\mathrm{RH} 1$ & $\mathrm{RH} 1$ & GL343273.1 & 0.64 \\
\hline & & Aca $4 \mathrm{RH} 2$ & $\mathrm{RH} 2$ & 4 & 122.81 \\
\hline & & Aca SWS1 & SWS1 & GL343828.1 & 0.11 \\
\hline & & Aca 2 sWS2 & SWS2 & 2 & 88.63 \\
\hline \multirow[t]{4}{*}{ Amphibians } & Western clawed frog & Xtr opn1lw & LWS & GL172911.1 & 0.21 \\
\hline & (JGI_4.2) & Xtr rho & $\mathrm{RH} 1$ & GL172832.1 & 1.64 \\
\hline & & Xtr opn1sw & SWS1 & GL173116.1 & 0.61 \\
\hline & & Xtr SWS2 & SWS2 & GL172911.1 & 0.22 \\
\hline \multirow[t]{3}{*}{ Coelacanths } & Coelacanth & Lch RH1 & $\mathrm{RH} 1$ & $\mathrm{JH} 126975.1$ & 0.80 \\
\hline & (LatCha1) & Lch RH2 & $\mathrm{RH} 2$ & $J H 126819.1$ & 1.63 \\
\hline & & Lch SWS2 & SWS2 & $J H 127263.1$ & 0.54 \\
\hline \multicolumn{6}{|l|}{ Actinopterygians } \\
\hline \multirow[t]{7}{*}{ Holostean fishes } & Spotted gar & Loc LG1 LWS & LWS & LG1 & 2.61 \\
\hline & (LepOcu1) & Loc LG5 RH1-1 ${ }^{d}$ & $\mathrm{RH} 1$ & LG5 & 40.45 \\
\hline & & Loc LG5 RH1-2 & $\mathrm{RH} 1$ & LG5 & 23.74 \\
\hline & & Loc LG3 RH2 & $\mathrm{RH} 2$ & LG3 & 36.55 \\
\hline & & Loc LG8 SWS1-1 ${ }^{\mathrm{e}}$ & SWS1 & LG8 & 10.39 \\
\hline & & Loc LG8 SWS1-2 ${ }^{d}$ & SWS1 & LG8 & 10.40 \\
\hline & & Loc LG1 SWS2 & SWS2 & LG1 & 2.62 \\
\hline \multirow[t]{5}{*}{ Teleost fishes } & Zebrafish & Dre 11 opn1lw1 & LWS & 11 & 26.41 \\
\hline & 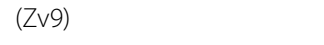 & Dre 11 opn1lw2 & LWS & 11 & 26.41 \\
\hline & & Dre exorh & $\mathrm{RH} 1$ & Zv9_NA986 & $0.130 \mathrm{~Kb}$ \\
\hline & & Dre 8 rho & $\mathrm{RH} 1$ & 8 & 55.71 \\
\hline & & Dre $11 \mathrm{rhol}$ & $\mathrm{RH} 1$ & 11 & 19.52 \\
\hline
\end{tabular}


Table 1 Summary of visual opsin sequences analyzed in this study (Continued)

\begin{tabular}{|c|c|c|c|c|c|}
\hline & & Dre 6 opn1mw1 & $\mathrm{RH} 2$ & 6 & 41.11 \\
\hline & & Dre 6 opn1mw2 & $\mathrm{RH} 2$ & 6 & 41.12 \\
\hline & & Dre 6 opn $1 \mathrm{mw} 3$ & $\mathrm{RH} 2$ & 6 & 41.12 \\
\hline & & Dre 6 opn1mw4 & $\mathrm{RH} 2$ & 6 & 41.13 \\
\hline & & Dre 4 opn 1 sw1 & SWS1 & 4 & 12.64 \\
\hline & & Dre 11 opn1sw2 & SWS2 & 11 & 26.41 \\
\hline & Three-spined stickleback & Gac XVII LWS & LWS & $X V I I$ & 10.63 \\
\hline & (BROADS1) & Gac XII RH1-1 d,e & $\mathrm{RH} 1$ & XII & 1.18 \\
\hline & & Gac XII RH1-2 & $\mathrm{RH} 1$ & $X I I$ & 0.81 \\
\hline & & Gac RH2-1 & $\mathrm{RH} 2$ & scaffold 27 & 4.15 \\
\hline & & $\mathrm{Gac} \mathrm{RH}_{2}-2$ & $\mathrm{RH} 2$ & scaffold 27 & 4.16 \\
\hline & & Gac SWS1 & SWS1 & scaffold 90 & 0.46 \\
\hline & & Gac XVII SWS2 & SWS2 & $X V I I$ & 10.62 \\
\hline & Medaka & Ola 5 LWS-1 & LWS & 5 & 27.02 \\
\hline & (MEDAKA1) & Ola 5 LWS-2 & LWS & 5 & 27.01 \\
\hline & & Ola 7 RH1-1 & $\mathrm{RH} 1$ & 7 & 17.43 \\
\hline & & Ola $7 \mathrm{RH} 1-2$ & $\mathrm{RH} 1$ & 7 & 17.10 \\
\hline & & Ola $\mathrm{RH}_{2}-1$ & $\mathrm{RH} 2$ & ultracontig 62 & 1.61 \\
\hline & & Ola RH2-2 & $\mathrm{RH} 2$ & ultracontig 62 & 1.62 \\
\hline & & Ola RH2-3 & $\mathrm{RH} 2$ & ultracontig 62 & 1.62 \\
\hline & & Ola SWS1 & SWS1 & scaffold 1021 & 0.04 \\
\hline & & Ola 5 SWS2-1 & SWS2 & 5 & 27.01 \\
\hline & & Ola 5 SWS2-2 & SWS2 & 5 & 27.00 \\
\hline & Spotted green pufferfish & Tni 11 LWS & LWS & 11 & 10.12 \\
\hline & (TETRAODON8) & Tni 9 RH1-1 & $\mathrm{RH} 1$ & 9 & 6.68 \\
\hline & & Tni 9 RH1-2 & $\mathrm{RH} 1$ & 9 & 6.48 \\
\hline & & Tni $11 \mathrm{RH} 2$ & $\mathrm{RH} 2$ & 11 & 5.97 \\
\hline & & Tni 11 SWS2 & SWS2 & 11 & 10.12 \\
\hline \multicolumn{6}{|c|}{ Cyclostomes } \\
\hline \multirow[t]{5}{*}{ Lampreys } & Pouched lamprey & Gau Lws & LWS & & \\
\hline & & Gau RhA & $\mathrm{RH} 1$ & & \\
\hline & & Gau RhB & $\mathrm{RH} 2$ & & \\
\hline & & Gau Sws1 & SWS1 & & \\
\hline & & Gau Sws2 & SWS2 & & \\
\hline
\end{tabular}

${ }^{\mathrm{a}}$ For the sequence names, species abbreviations are applied as described in Methods, followed by the number of the chromosome or linkage group where the gene is located (if known) and the gene/subtype name. Where available, gene names follow approved symbols by the HUGO Gene Nomenclature Committee and interacting committees, accessed via www.genenames.org, and the Zebrafish Model Organism Database (ZFIN), accessed via www.zfin.org. Geotria australis gene names follow [36]. ${ }^{b}$ Subtype designations follow the tree topology presented in Figure 1.

In the chicken genome assembly available in the Ensembl genome browser (WASHUC2) only two of the five ancestral visual opsin types were identified. However the three missing genes have previously been cloned and are available in the NCBI GenBank database (see Additional file 1).

dSequences manually predicted de novo (see Methods).

eSequence predictions are fragments and do not span the whole length of the alignment.

consisted of five visual opsin genes with losses of SWS2 and $R H 2$ in mammals. The Western clawed frog (Xenopus tropicalis) also seems to have lost the $R H 2$ gene, although this could be due to gaps in the genome assembly. Three full-length and one fragmented visual opsin gene sequences were identified in the coelacanth (Latimeria chalumnae) genome assembly. The full-length sequences cluster within the RH1, RH2 and SWS2 branches (Figure 1). The RH1 and RH2 sequences have previously been reported [38]. The presence of only three visual opsin sequences indicates that there seem to have been losses of visual opsin genes in this lineage. The fragmented gene 




Figure 1 Phylogenetic relationships between the visual opsin genes of the LWS, SWS1, SWS2, RH1 and RH2 clades. Tree topology inferred with the phylogenetic maximum likelihood method from an amino acid sequence alignment, supported by a non-parametric bootstrap analysis with 100 replicates. Red arrowheads indicate nodes with bootstrap values lower than $50 \%$ that were not considered informative. The tree is rooted with the human OPN3 sequence (not shown). Inlaid: (A) Neighbor joining (NJ) topology of the LWS clade, (B) NJ topology of the RH1 clade. See Additional file 2: Figures S1 and S2 for full trees, including all bootstrap values and root. For the sequence names, species abbreviations are applied as described in Methods, followed by the number of the chromosome or linkage group where the gene is located (if known) and the gene/subtype name (see Table 1). Scale bars indicate phylogenetic distance as number of substitutions per site.

sequence appears to be a pseudogene sharing sequence similarity to known SWS1 sequences, but with a premature stop-codon and a frame-shift mutation within the first exon. A sequence fragment bearing the same mutations at the same locations has been identified previously [38] as one of two non-overlapping clones postulated to 
be parts of an SWS1 pseudogene. However, the second clone reported by these authors, instead corresponds to a fragment of the full-length $S W S 2$ sequence that we identified. All five ancestral types of vertebrate visual opsin genes are present in all the investigated actinopterygian genomes (including spotted gar and teleosts), except the spotted green pufferfish, where an SWS1 sequence could not be identified (Figure 1). Additionally, LWS, SWS2, RH1 and RH2 sequences often occur as multiple local duplicates in teleosts (Table 1). In the spotted gar genome assembly we could identify seven visual opsin genes: one gene each of the LWS, SWS2 and $R H 2$ types and two genes each of the SWS1 and RH1 types (Figure 1). The two SWS1 genes are located adjacent to each other on the same linkage group (LG8) approximately $8 \mathrm{~Kb}$ apart (see Table 1 ), and are thus most likely the result of a local duplication. The two $R H 1$ genes are located on the same linkage group (LG5) approximately $16.6 \mathrm{Mb}$ apart, one with introns (RH1-1 in Table 1 and Figure 1) and one without (RH1-2). These duplicate $R H 1$ genes on the same chromosome have previously been identified in teleost fish species: the one with introns, called exo-rhodopsin, is expressed outside of the retina; and the one without introns, called rhodopsin, is expressed in rods [39]. The intron-less rhodopsin gene is the result of a retrotranscription event [40]. We could identify these duplicate $R H 1$ sequences in all investigated teleost genomes (RH1-1 and RH1-2 in Table 1 and Figure 1). Additionally, the zebrafish has two copies of the intron-less rhodopsin gene, located on two different chromosomes. These zebrafish genes have previously been called rh1 and rh1-2 [41] or rho and rhol (for rhodopsin-like) (Table 1). The teleost exo-rhodopsins form a well-supported cluster in the phylogenetic analyses (marked exorh in Figure 1) while the putative spotted gar exo-rhodopsin (RH1-1) has an uncertain position within the RH1 cluster. On the other hand, the rhodopsin gene of the spotted gar clusters together with the teleost rhodopsin genes with high support, forming a well-defined actinopterygian cluster (marked rho in Figure 1).

Five visual opsins have previously been cloned and characterized from the pouched lamprey (Geotria australis) [36] and were therefore included in our phylogenetic analyses to provide relative dating. In line with previous analyses $[2,7,36]$ the pouched lamprey LWS, SWS1 and SWS2 sequences cluster with high support within their respective branches (Figure 1). We also find that the sequences called $R h A$ and $R h B$ represent the RH1 and $R H 2$ genes, with high support in all our phylogenetic analyses (Figure 1, Additional file 2). Thus it is likely that all five visual opsin genes were present before the divergence of cyclostomes, such as the lampreys, and jawed vertebrates.

\section{Phylogenetic analysis of the oxytocin and vasopressin receptor genes}

We have updated recent phylogenetic analyses of the OT/VP-R gene family [29,30] by including sequences from the coelacanth, spotted gar and Southern platyfish. This was done in order to improve the taxonomic representation and complement our conserved synteny analysis, which includes positional data from the spotted gar genome assembly. Both NJ and PhyML trees were made (Additional file 2: Figures S5 and S6), and the PhyML tree is shown in Figure 2. Our updated phylogenetic analyses are consistent with the previously cited literature, but also show several new results.

The clusters for oxytocin receptors (OTR) and V1-type vasopressin receptors (V1A, V1B) are well-supported, and the tree shows that the spotted gar, like teleosts, lacks the $V 1 B$ subtype while the coelacanth has all three (Figure 2). With regard to V2-type vasopressin receptors, Yamaguchi et al (2012) were able to define three types by using synteny data [29]. Our phylogenetic analyses and the analysis of conserved synteny described below are consistent with three ancestral V2-types, and we propose the nomenclature $V 2 A, V 2 B$ and $V 2 C$ for these receptors. As in the previously cited analyses, the $V 2 C$ receptors form two paraphyletic branches, with teleost $V 2 C$ sequences clustering basal to the other V2-type branches (Figure 2), likely due to a faster rate of sequence evolution. However, their chromosomal locations support their orthology with tetrapod and spotted gar $V 2 C$ sequences (see below). The coelacanth lacks a $V 2 C$ sequence but has both $V 2 A$ and $V 2 B$, making it the only analyzed species in the sarcopterygian lineage (which also includes tetrapods) with a $V 2 B$ gene. Since $V 2 C$ sequences were found in the Western clawed frog, anole lizard and chicken genomes (Figure 2), the loss in the coelacanth is likely lineage-specific. Taken together with the spotted gar, which has all three V2-type sequences (Figure 2), this indicates that the $V 2 A, V 2 B$ and $V 2 C$ subtypes arose early in vertebrate evolution, although there have been several differential losses in different vertebrate classes. Sequences from the Southern platyfish were included since this species was found to have the $V 2 C$ sequence that previously had only been found in zebrafish and three-spined stickleback [30]. These three species do not form a monophyletic group within teleosts, which indicates that $V 2 C$ genes could have been lost several times in teleost evolution. The Southern platyfish is also the only teleost where duplicate $V 2 B$ sequences were found ( $V 2 B-1$ and $V 2 B-2$ in Figure 2 ).

Database identifiers, location data, genome assembly information, and annotation notes for all identified OT/ VP- $R$ sequences are included in Additional file 1. The OT/VP-R phylogenetic tree files and the alignment they were made from are provided as a citable file set with a stable identifier - see reference [42]. 


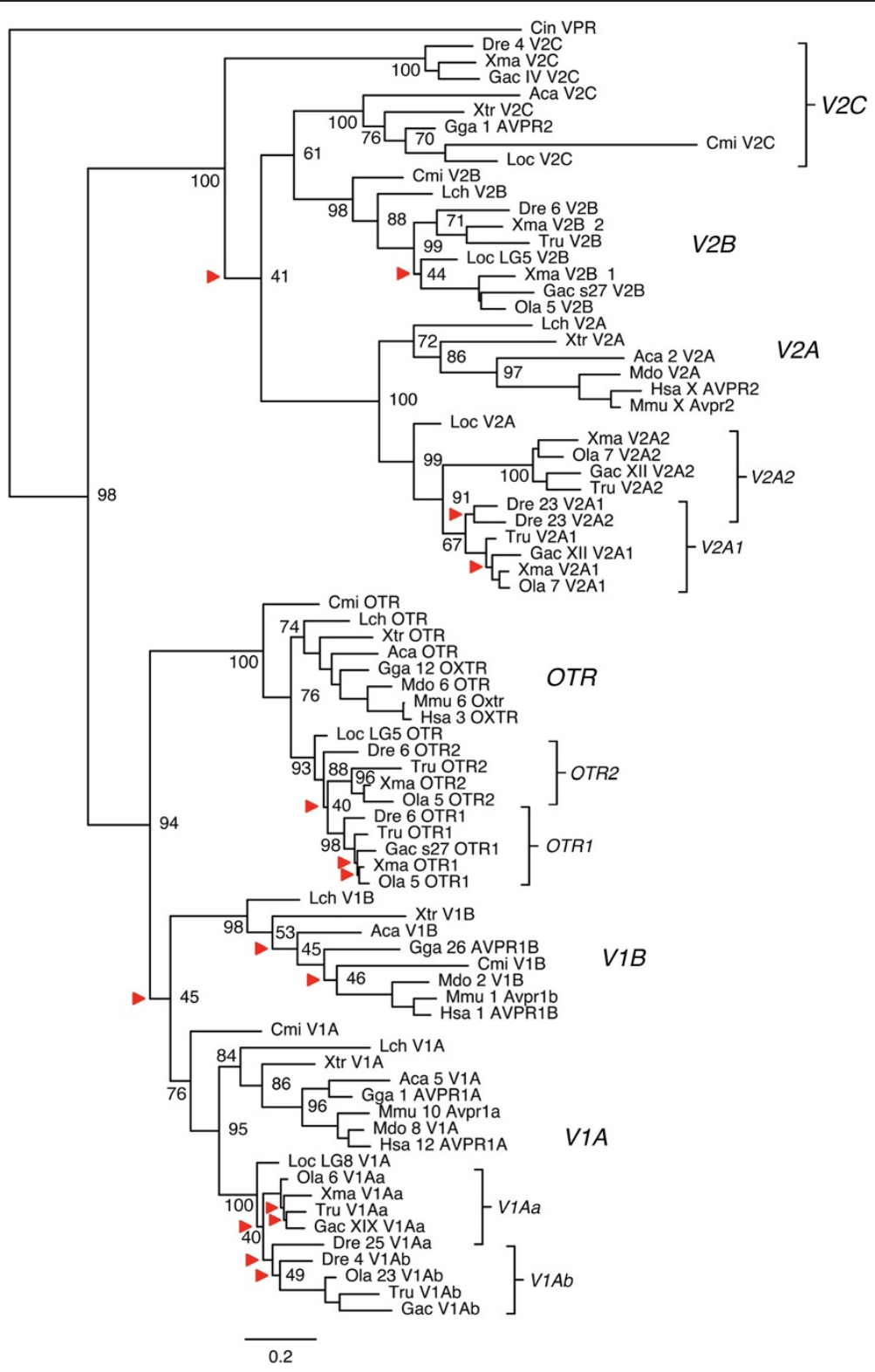

Figure 2 Phylogenetic relationships between oxytocin and vasopressin receptor subtype genes. Tree topology inferred with the phylogenetic maximum likelihood method from an amino acid sequence alignment, supported by a non-parametric bootstrap analysis with 100 replicates. Arrowheads indicate nodes with bootstrap values lower than $50 \%$ that were not considered informative. Rooted with the common octopus OTR, CTR1 and CTR2 sequences (not shown). See Additional file 2: Figures S5 and S6 for full trees with all bootstrap values and root, including a neighbor joining topology. Sequence names and scale as in as in Figure 1. Teleost fish duplicates are indicated by brackets. The V2C sequences do not form a well-supported clade; this is also indicated with a bracket. Approved gene names are used for human, mouse and chicken genes, otherwise subtype names are used. Some of the sequence predictions used to make the tree are fragments and do not span the whole length of the alignment (see Additional file 1).

Phylogenetic analysis of the L-type voltage-gated calcium channel alpha subunits

Four genes of the CACNA1-L family were identified in the tetrapod genomes investigated: CACNA1D, CAC$N A 1 F, C A C N A 1 C$ and CACNA1S. However CACNA1F could not be identified in the chicken genome assembly or in any other avian genome available. The Western clawed frog was excluded from the analysis because the short scaffolds of the genome assembly (JGI4_1) did not allow complete gene sequences to be identified. In the teleost genomes investigated all four genes are present, with additional putative $3 \mathrm{R}$ duplicates of the $C A C N A 1 D,-1 F$ and $-1 S$ genes in all four teleost genomes. These results are detailed in Additional file 1, with database identifiers, location data, genome assembly information, and annotation notes for all identified sequences. 
The CACNA1F, $-1 C$ and $-1 S$ genes form three distinct clusters in the bootstrapped NJ (Additional file 2: Figure S7) and PhyML (Figure S8) analyses, while the putative teleost CACNA1D subtype cluster is only resolved in the NJ tree (Additional file 2: Figure S7). These results are presented in Additional file 2. All CACNA1-L phylogenetic tree files and the alignment they were made from are provided as a citable file set with a stable identifier - see reference [43].

\section{Conserved synteny analysis}

In total, 41 neighboring gene families showed patterns of conserved synteny in the chromosomal regions harboring members of the five main gene families. Seven of the 41 gene families were discarded upon preliminary analyses because their multitude of members in the genome databases made phylogenetic analyses unreliable, or because their topologies could not be resolved, leaving 34 gene families in our final dataset. These families are summarized in Table 2. The locations of the identified genes were recorded for 12 species, representing five of the vertebrate classes, with available genome assemblies (see Conserved synteny analysis in Methods), and phylogenetic analyses using neighbor joining (NJ) and phylogenetic maximum likelihood (PhyML) methods were carried out for each gene family in order to determine orthology and paralogy relationships. Database identifiers, location data and annotation notes for the neighboring gene families, including those that were discarded, are provided in Additional file 3 and all corresponding alignments and phylogenetic trees are provided as a citable file set with a stable identifier - see reference [44].

For the majority of the identified neighboring gene families, 23 out of 34, both the NJ trees and the PhyML trees support duplications in the same time-window as the $2 \mathrm{R}$ events, i.e. after the divergence of invertebrate chordates and vertebrates, but before the divergence of sarcopterygians and actinopterygians. Several of the 34 identified families also have duplicate teleost branches supporting duplications in the same time-window as the teleost-specific 3R event. Four additional gene families are consistent with duplications in $2 \mathrm{R}$, but lack invertebrate family members to date the duplications. The remaining seven gene families have some subtype clusters that are unresolved, or an unresolved branching of the identified tunicate or lancelet sequences, which makes them inconclusive with regard to the duplications in 2R. Detailed notes on the tree topologies for the neighboring gene families are provided in Additional file 4 .

To investigate the involvement of the whole genome duplications, the chromosomal locations of all neighboring family members were recorded and compared across species. A compiled list of comparisons between the human, chicken, zebrafish and three-spined stickleback chromosome regions is shown in Additional file 5. This dataset shows the conserved synteny between the visual opsin gene-bearing chromosome regions in the analyzed genomes, which also includes the OT/VP-R, CACNA1-L and GNAT/GNAI gene families. These paralogous chromosome regions correspond to blocks present on human chromosomes 1, 3, 7, 12 and $\mathrm{X}$, and chicken chromosomes 1, 12 and 26 (Figure 3). The blocks on human chromosomes 7 and 12 seem to be the result of a linkage break in the human lineage, since the chicken orthologs of the genes in both of these blocks are all located on chicken chromosome 1 . This is supported by our orthology analysis of the spotted gar genome (Additional file 5). Notably, no chicken orthologs of the genes located on the human $\mathrm{X}$ chromosome were found in the chicken genome database, indicating that this whole segment of the chicken genome is missing from the genome assembly (WASHUC2, latest accessed on Ensembl 71, Apr 2013).

The teleost-specific duplication of whole chromosome regions is evident in the teleost genomes for the regions harboring OPN1SW,V1A, CACNA1C and GNAI1 genes (Figure 4). These regions of chicken chromosome 1 and human chromosomes 7 and 12 correspond to blocks on zebrafish chromosomes 4 and 25 (Figure 4), and threespined stickleback linkage groups IV and XIX (Additional file 5), with several of the gene families having teleostspecific duplicates in both chromosome blocks. However, our analyses also demonstrate that there have been extensive rearrangements in the teleost genomes, which obscure the involvement of both the $2 \mathrm{R}$ and $3 \mathrm{R}$ events. For instance, genes located on the chromosomes 1 and 3 and the $\mathrm{X}$ chromosome in the human genome have orthologs distributed between zebrafish chromosomes $6,8,11$ and 23 in a way that suggests translocation of paralogous genes between these chromosome regions after 3R (Figure 4 and Additional file 5).

In order to date the translocations seen in the teleost genomes better, orthology predictions between the human neighboring family members and the spotted gar genome were made (see Methods). The locations of the predicted spotted gar orthologs were then recorded and compared with the human, chicken, zebrafish and three-spined stickleback chromosome regions (Additional file 5). While only around $75 \%$ of putative spotted gar orthologs could be found for the human neighboring gene family members, this analysis of conserved synteny between the human and spotted gar shows no translocations in the spotted gar genome. With a few exceptions, the identified putative spotted gar orthologs are located on linkage groups 1, 3 and 5, which correspond to the regions on human chromosomes $\mathrm{X}, 1$ and 3 respectively, and linkage group 8 which corresponds to the regions on human chromosomes 7 and 12 (Figure 3). 
Table 2 Neighboring gene families analyzed for conserved synteny

\begin{tabular}{|c|c|c|}
\hline Symbol & Description & $\operatorname{Root}^{1}$ \\
\hline ATP2B & ATPase, Ca++ transporting, plama membrane & \\
\hline B4GALNT & Beta-1,4-N-acetyl-galactosaminyl transferase & Midpoint \\
\hline CACNA2D & Calcium channel, voltage-dependent, alpha 2/delta subunit & \\
\hline CAMK1 & Calcium/calmodulin dependent protein kinase & \\
\hline CDK & Cyclin-dependent kinase, members 16,17 and 18 & C. elegans \\
\hline CELSR & Cadherin, EGF LAG seven-pass G-type receptor (flamingo homolog, Drosophila) & \\
\hline CNTN & Contactin precursor & \\
\hline COPG & Coatomer protein complex, subunit gamma & \\
\hline ERC & ELKS/RAB6-interacting/CAST family & \\
\hline FLN & Filamin & \\
\hline GXYLT & Glucoside xylosyltransferase & \\
\hline IKBKE & Kinase epsilon and TANK-binding kinase & \\
\hline IQSEC & IQ motif and Sec7 domain containing & \\
\hline KDM & Lysine specific demethylase 5 & \\
\hline KLHDC & Kelch domain containing 8 & C. intestinalis \\
\hline L1CAM & L1 cell adhesion molecule & \\
\hline LRRN & Leucine rich repeat neuronal & C. intestinalis \\
\hline MAGl & Membrane associated guanylate kinase, WW and PDZ domain containing & \\
\hline PHTF & Putative homeodomain transcription factor & C. savignyi \\
\hline PLG & Plasminogen ortholog & C. intestinalis \\
\hline PLXNA & Plexin A & \\
\hline PPM1 & Protein phosphatase, $\mathrm{Mg} 2+/ \mathrm{Mn} 2+$ dependent & \\
\hline PRICKLE & Prickle homolog & \\
\hline PTPN & Protein tyrosine phosphatase, non-receptor type & \\
\hline RBM & RNA binding motif protein & \\
\hline RSBN & Round spermatid basic protein & \\
\hline SEMA3 & Sema domain, immunoglobulin domain (Ig), short basic domain, secreted, (semaphorin) & Midpoint \\
\hline SRGAP & SLIT-ROBO Rho GTPase activating protein & C.intestinalis \\
\hline SYP & Synaptophysin & C.elegans \\
\hline TIMM17 & Translocase of inner mitochondrial membrane 17 & C.elegans \\
\hline TWF & Twinfilin & \\
\hline UBA & Ubiquitin-like modifier activating enzyme, members 1 and 7 & \\
\hline USP & Ubiquitin specific peptidase, members 4, 11, 15 and 19 & Midpoint \\
\hline WNK & WNK lysine deficient protein kinase & \\
\hline
\end{tabular}

${ }^{1}$ If other than Drosophila melanogaster.

\section{Discussion}

In order to investigate the evolution of the visual opsin genes related to the two rounds of early vertebrate whole genome duplications, $2 \mathrm{R}$, we have analyzed several neighboring gene families identified in the same chromosomal regions as the human visual opsin genes. Specifically, we investigated whether there were other gene families showing conserved synteny with these and whether they underwent gene duplications in the same time-window as the $2 \mathrm{R}$ events. During the process of these analyses we realized that the chromosomal regions of the visual opsin genes overlapped with similar ongoing analyses in our laboratory of the oxytocin and vasopressin receptor gene family (OT/VP-R), the G-protein alpha transducing subunit (GNAT) and G-Protein alpha inhibiting subunit (GNAI) gene families, as well as the gene family of L-type voltagegated calcium channel alpha subunits (CACNA1-L). These gene families are referred to as the "main" gene families in our analyses. Their evolution will be discussed below in conjunction with the evolution of the visual opsins. 


\section{Human}

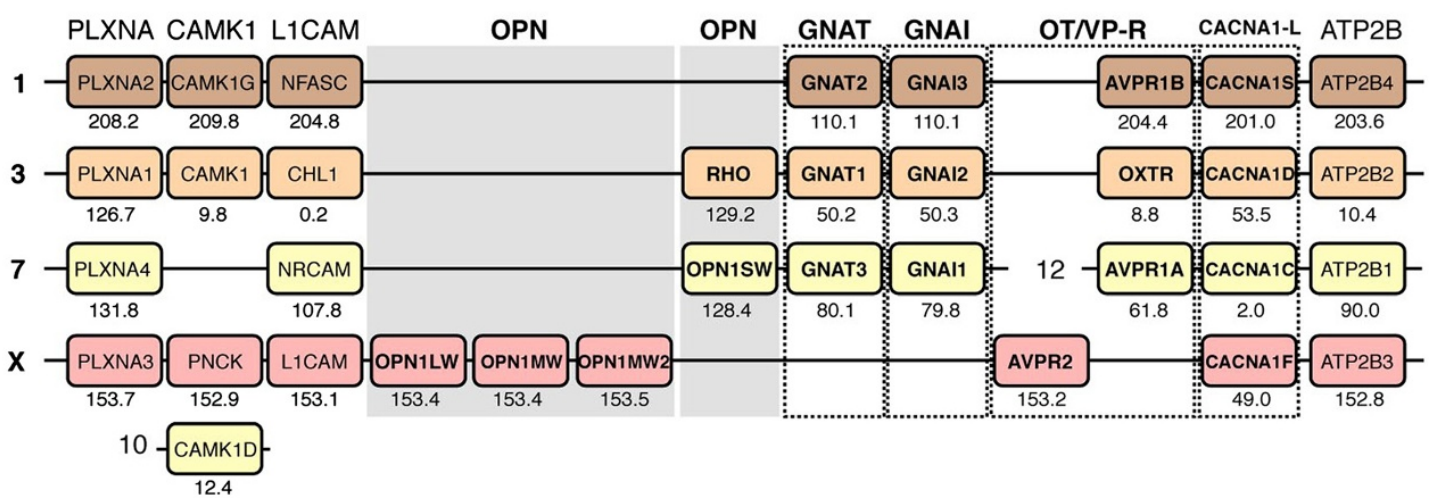

\section{Chicken}

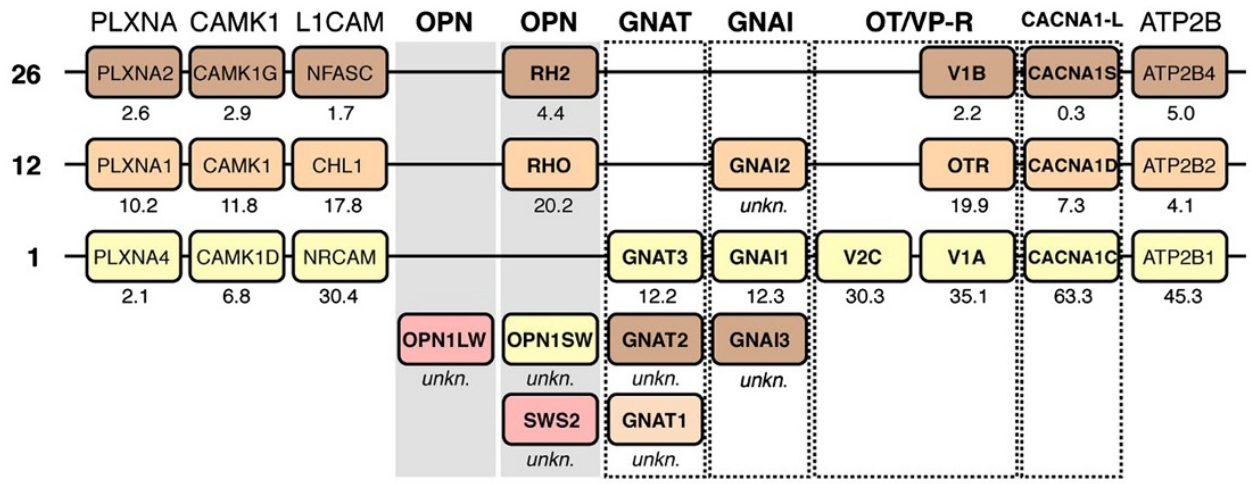

\section{Spotted gar}

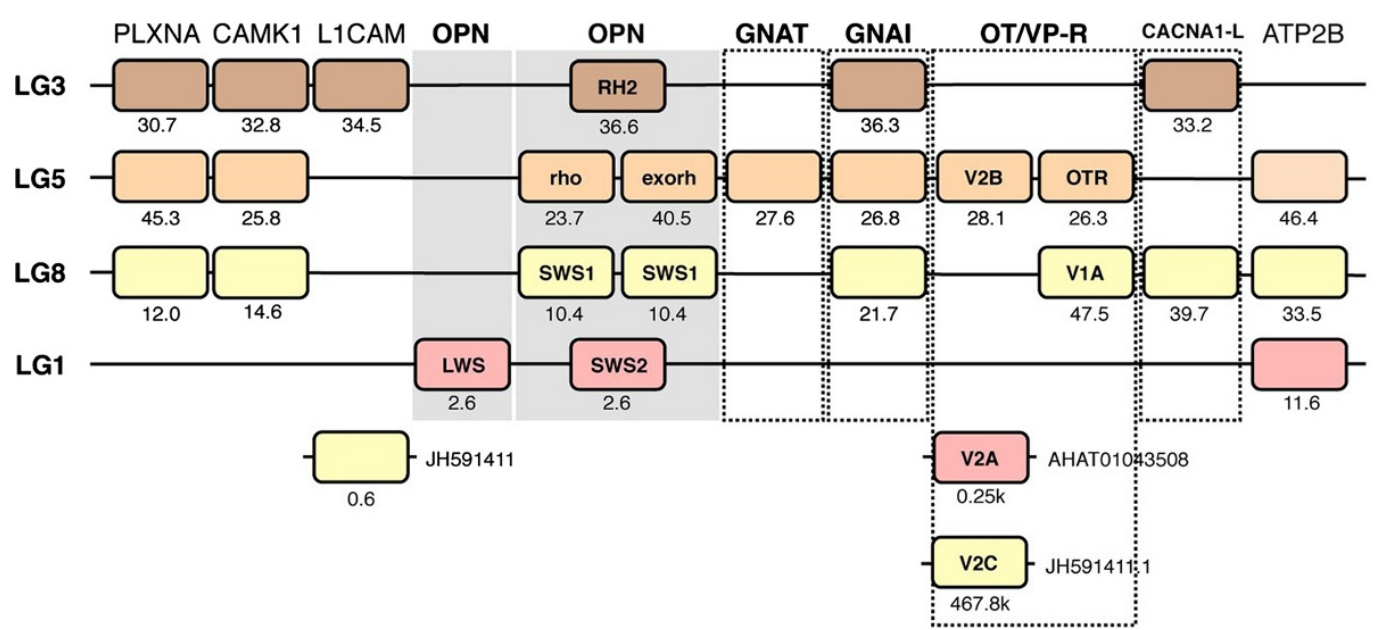

Figure 3 Conserved synteny between 2R-generated chromosome blocks. The identified paralogous chromosomal regions in the human, chicken and spotted gar genomes. In addition to the main gene families, the neighboring gene families with a full quartet of paralogous genes (ATP2B1, CAMK1, L1CAM and PLXNA) are included for reference. Colors are applied following the human and chicken chromosomes in order to show conserved synteny as well as sequence homology between species. Four paralogous regions can be observed in the human and spotted gar genomes. In the chicken genome the orthologs of the human genes on chromosome X could not be identified in the genome assembly. Orthologs for the genes on human chromosomes 7 and 12 are located on chromosome 1 in chicken and LG8 in spotted gar, indicating a split of this region in the human lineage. Several chicken genes have not been mapped to any chromosomal location. Their sequences for phylogenetic analyses were retrieved from NCBI. To facilitate comparisons between species, the names of the human orthologs have been applied to the chicken genes except for the visual opsin and OTNP-R families where the gene names used in Figures 1 and 2 are applied. Note that the human and chicken V2 receptor sequences correspond to different subtypes: V2A and V2C respectively (Figure 2). 


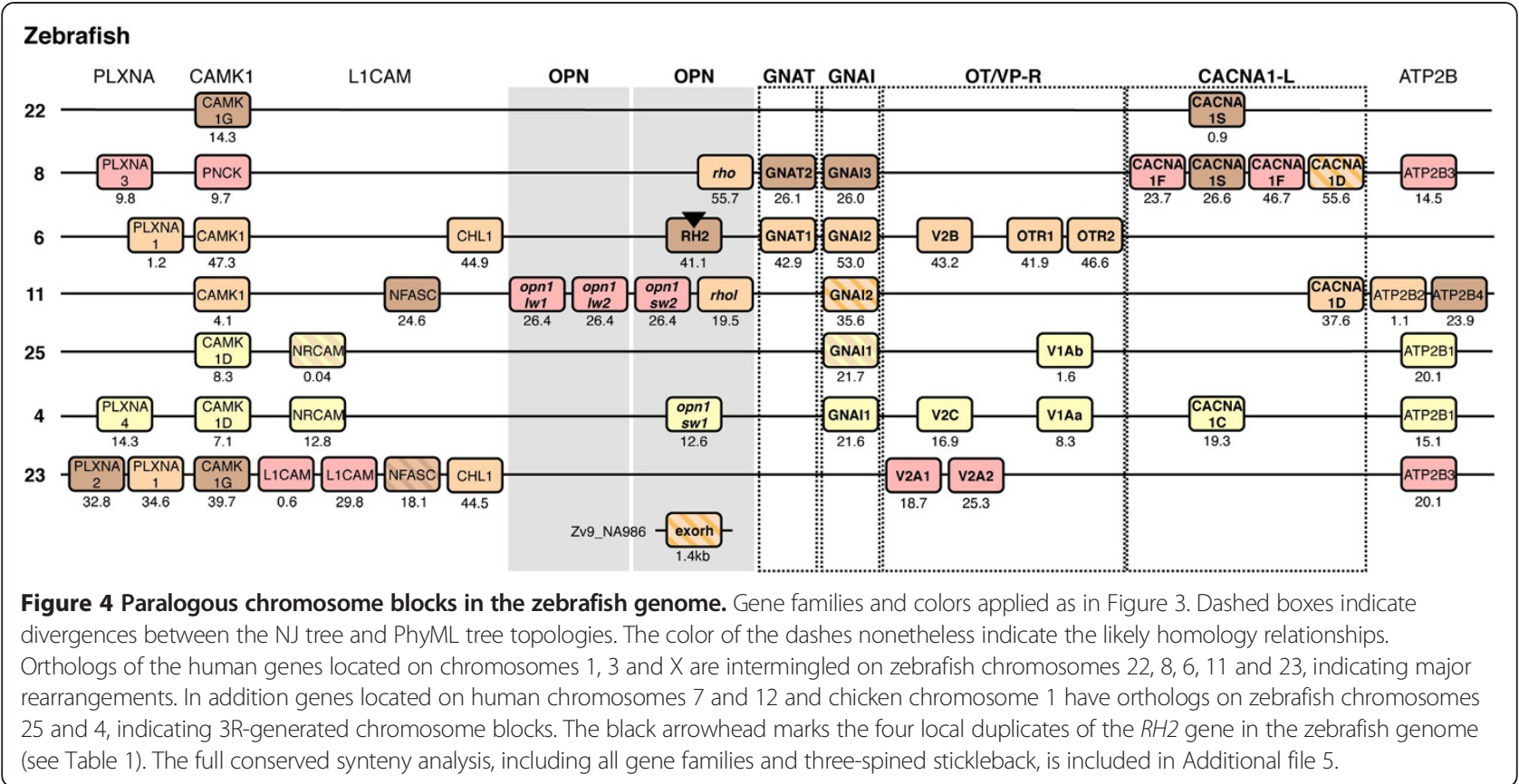

The chromosomal regions harboring the visual opsin genes were duplicated in the vertebrate whole genome duplications

In total, the chromosomal locations and phylogenetic analyses of 34 neighboring gene families have been analyzed. The orthology and paralogy relationships within each family were inferred and, using relative dating, the time-window for the expansion of the gene families was determined. The synteny data and phylogenetic analyses taken together show that the chromosome regions bearing the visual opsin genes are paralogous and were formed by chromosome duplications in the same time-window as the $2 \mathrm{R}$ events, after the divergence of invertebrate chordates and before the divergence of actinopterygians (including teleosts and spotted gar) and sarcopterygians (including tetrapods and coelacanth). Our analyses also show that the chromosomal regions were duplicated once more in early teleost evolution, consistent with the time-window of the $3 \mathrm{R}$ event. Our proposed evolutionary scenario for the evolution of these chromosome regions is presented in Figure 5. In the human genome, the paralogous chromosomal regions correspond to blocks of chromosomes 1, 3, 7, 12 and X (Figure 3), with the blocks on 7 and 12 together representing one of the four ancestral blocks. These genomic regions have previously been identified as part of a paralogon in large-scale genomic analyses $[10,11,45]$.

\section{Chromosomal rearrangements in teleost genomes, but not in the spotted gar genome}

In the teleost genomes the investigated orthologous genes of the human genes located on chromosomes 1, 3 and $\mathrm{X}$ seem to have been rearranged so that the genes are intermingled. In zebrafish this involves chromosomes 8, 23, 11 and 6 (Figure 4) and in the three-spined stickleback linkage groups XII, XVII and scaffold 27 (Additional file 5). These major rearrangements are in line with previous whole genome analyses [10,45]. Recently Amores et al. published an analysis of the genome of the spotted gar (Lepisosteus oculatus), which belongs to a actinopterygian lineage that diverged before $3 \mathrm{R}$ and thus should not have the same rearrangements [46]. They found that the spotted gar seemed to have fewer rearrangements in general compared to the teleosts and that the synteny was more conserved between human and spotted gar than between spotted gar and zebrafish due to post-3R rearrangements in the teleost lineage. To see if this was the case for the regions housing the visual opsin genes, we performed an orthology prediction between human and spotted gar protein predictions and recorded the chromosomal locations of the putative spotted gar orthologs (Additional file 5). This is summarized in Figure 3. By comparing the chromosomal regions of human, chicken, spotted gar, zebrafish and three-spined stickleback we found that the synteny is indeed more conserved between human, chicken and spotted gar than between spotted gar and the teleosts, in agreement with the global genome analyses [46]. This further supports the notion that $3 \mathrm{R}$ contributed to the major rearrangements seen in teleost genomes $[10,45]$.

\section{Evolution of the visual opsin gene family}

Our phylogenetic analyses and the analyses of conserved synteny of the visual opsins and their chromosomal 
regions support the evolutionary scenario previously proposed by us [22,23] where a local duplication occurred before $2 \mathrm{R}$ giving rise to two ancestral genes, $S W S$ and $L W S$. These genes later duplicated in $2 \mathrm{R}$ as part of a larger chromosomal block so that the ancestral SWS gene gave rise to the types $S W S 1, S W S 2, R H 1$ and $R H 2$ and the ancestral LWS gave rise to four copies out of which only one has been retained, namely the $L W S$ type (Figure 5). Although the pinopsins and vertebrate ancient (V/A) opsins cluster with the visual opsins in phylogenetic trees (Additional file 2: Figures S3 and S4), the present synteny analysis does not indicate that pinopsins and V/A opsins arose in the same chromosome duplications that gave rise to the visual opsin gene family. The current repertoire of visual opsin genes in vertebrates, and their evolution, is summarized in Figure 6.

Five of the seven identified spotted gar genes represent the LWS, SWS1, SWS2 and RH2 types of visual opsin genes, while the other two represent the RH1 type (Figure 1): one intron-containing (RH1-1) and one 
intron-less (RH1-2). The intron-containing $R H 1$ gene is likely the ortholog of the teleost exo-rhodopsin gene (exorh), named for its expression mainly in the pineal complex of the teleost brain instead of the retina [39]. The intron-less $R H 1$ gene is likely the ortholog of the teleost rhodopsin (rho) gene, a retrotranscript of the exo-rhodopsin gene [47]. Our analyses indicate that the retrotransposition event occurred before the divergence of holostean fishes (including gars) and teleosts (Figure 6). The intron-less spotted gar $R H 1$ is presumably the ortho$\log$ of a gene that has previously been identified in the longnose gar (Lepisosteus osseus) as rhodopsin [48]. In the zebrafish there is an additional intron-less $R H 1$ gene called rhol for rhodopsin-like (Figure 2), located on a different chromosome than the rho gene (Table 1). Morrow et al., who identified the two intronless RH1 genes in the zebrafish genome, also identified similar duplicated genes in a few other cyprinid species [41]. Analyses performed by other researchers have shown that duplicated intron-less $R H 1$ genes seem to be present in some non-cyprinid teleost species as well, including the deep-sea dwelling short-fin pearl-eye [49] and the Japanese and European eels [50,51]. This suggests that the two intron-less $R H 1$ genes might have originated in $3 \mathrm{R}$, following the retrotransposition event. However, to confirm that rho and rhol are 3R duplicates, information about chromosomal locations is needed from several species. The presence of two intronless RH1 genes in the above-mentioned species, but not in the medaka, spotted green pufferfish and three-spined stickleback, suggests loss in the latter species or in their common ancestor. In the zebrafish, the regions harbouring the duplicated $R H 1$ genes rho and rhol on chromosome 8 and 11 have undergone major rearrangements. Our analyses show that these chromosomes contain paralogous members of the CACNA1-L, ERC, CACNA2D, PRICKLE and MAGI families (see Additional file 5). The CACNA2D and MAGI tree topologies support a 3R duplication of these paralogous gene copies. However, the paralogous copies in the trees of the CACNA1-L, ERC and PRICKLE families are inconsistent. While it is a likely scenario, we cannot say for certain that the duplicate intron-less $R H 1$ genes originated in $3 R$.

\section{Evolution of the GNAT and GNAI gene families}

In our previous analyses of the GNAT gene family we concluded that the GNAT-GNAI gene pairs seen today in many vertebrates had an origin in a local duplication preceding $2 \mathrm{R}$. The $2 \mathrm{R}$ events subsequently gave rise to the GNAT1-GNAI2, GNAT2-GNAI3 and GNAT3-GNAI1 gene pairs [21]. This is in line with previous studies from other research groups, see for instance [27]. Consequently, independent losses of the GNAT3 gene have occurred in the teleost and amphibian lineages and possibly the sea lamprey (Petromyzon marinus) lineage after 2R $[27,28]$. We identified putative 3R duplicates in the GNAI gene family for the GNAI1 and GNAI2 genes in our previous analysis [21], although we had no synteny data to support the hypothesis. In the present analyses 10 gene families have putative 3R duplicates located on the GNAI1-bearing chromosomes in zebrafish (chromosomes 4 and 25). Six of these families show a clear 3R topology in both
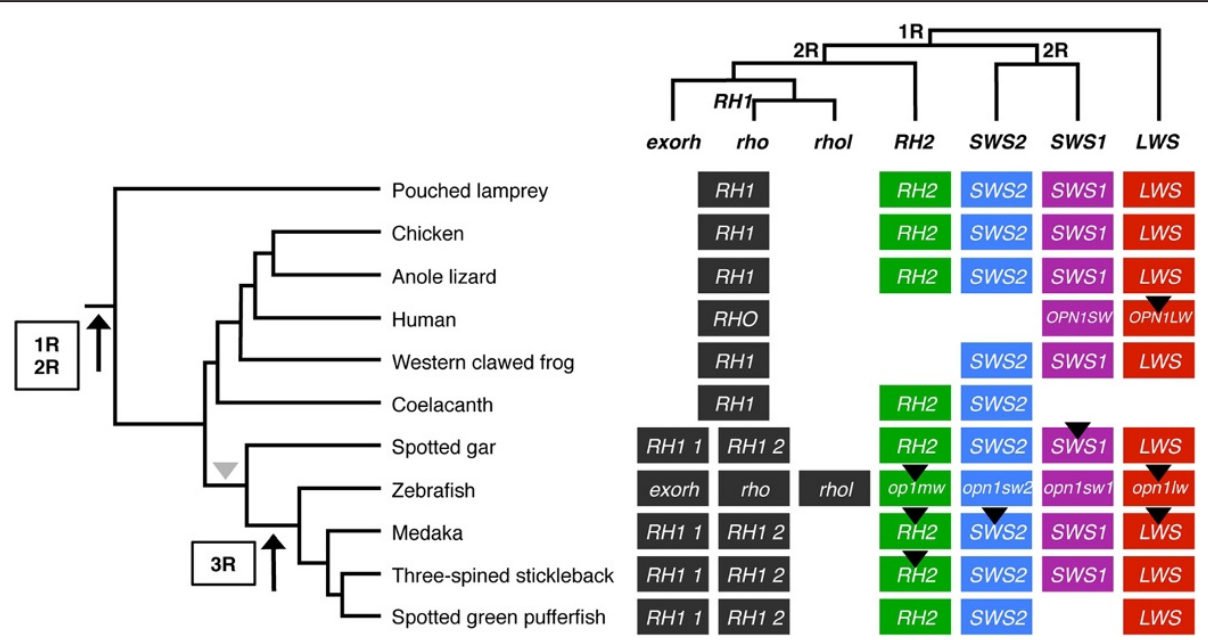

Figure 6 Visual opsin gene repertoires in vertebrates. The tree to the left shows the evolutionary relationship between species used in our analyses, with the time-windows for the $2 \mathrm{R}$ and $3 \mathrm{R}$ events. The upper tree shows the relationship between LWS, SWS1, SWS2, RH1 and RH2 visual opsins inferred from phylogenetic analyses and synteny data. Black arrowheads mark the presence of local duplicates. The retrotransposition event that gave rise to intron-less rhodopsin (RH1) genes in spotted gar and teleosts is marked with a grey arrowhead in the left panel. The intron-less RH1 genes are called rho and rhol, and exorh denotes the exo-rhodopsin genes, using the approved zebrafish names. For human and zebrafish genes, the approved gene names are used as indicated in footnote a of Table 1. 
phylogenetic analyses. In the three-spined stickleback 12 neighboring gene families have members on the GNAI1-bearing chromosomes (IV and XIX), seven of which show a clear 3R topology in both phylogenetic analyses. In addition, three of the 10 families in zebrafish as well as three out of 12 families in three-spined stickleback show a topology supporting 3R in either the NJ or PhyML tree. For the GNAI2 genes, four neighboring gene families have putative 3R duplicates on the GNAI2 bearing chromosomes in zebrafish (chromosomes 6 and 11). Three of these show a clear $3 R$ duplication pattern. In three-spined stickleback only one family has members on the GNAI2 bearing chromosome and scaffold (XVII and scaffold 27), although its topology is unclear with regard to 3R. These results are summarized in Additional file 5. Our orthology predictions between human and spotted gar identified a single putative GNAT1 ortholog as well as a single putative GNAI1-3 ortholog (Figure 3). Taken together this analysis supports a possible 3R expansion of the GNAI1 and GNAI2 genes and their chromosomal regions. This also corroborates the loss of the GNAT3 gene in the teleost and amphibian lineages independently.

\section{Evolution of the oxytocin and vasopressin receptor gene family}

Our phylogenetic analysis of the OT/VP-R family (Figure 2) shows that the vertebrate gene family consists of six ancestral members, OTR, V1A, V1B, V2A,V2B and V2C. We propose a simplified nomenclature for the V2-type receptors based on the evolutionary scenario below. The $V 2 A$ receptors form the largest branch and include the well-characterized mammalian V2 receptor encoded by the $A V P R 2$ gene. The $V 2 B$ receptor subtype was identified independently by us [30] and other researchers [29], and includes mostly actinopterygian sequences as well as coelacanth and elephant shark. The $V 2 C$ receptor subtype was first reported by us as V2-like in zebrafish and three-spined stickleback [30], and it was later characterized as V2bR2 by Yamaguchi et al. [29]. Unlike V2B, the $V 2 C$ branch includes frog and lizard sequences, as well as the known chicken $\mathrm{V} 2$ receptor first characterized as VT1 [52]. Like the receptors OTR, V1A and V1B, both $V 2 B$ and $V 2 C$ seem to signal via the DAG/IP3/ $\mathrm{Ca}^{2+}$ pathway, while the adenylyl cyclase/PKA/cAMP signaling typical for $V 2 A$ receptors seems to constitute an evolutionary switch in the OT/VP-R family [29].

Our current phylogenetic analysis and the analyses of the visual opsin gene-bearing chromosome regions allowed us to deduce the following evolutionary scenario for the OT/VP-R gene family: Two ancestral genes were present on the same ancestral vertebrate chromosome before $2 \mathrm{R}$, one giving rise to $V 1 A, V 1 B$ and $O T R$ through $2 \mathrm{R}$ and one giving rise to $V 2 \mathrm{~A}, V 2 B$ and $V 2 C$ (Figure 5). The ancestral linkage is still conserved with $V 2 B$ and $O T R$ genes located together, and $V 1 A$ and $V 2 C$ genes located together on the same chromosomes. In the teleost lineage, the $3 \mathrm{R}$ event gave rise to two copies of $V 1 A$, called $V 1 A a$ and $V 1 A b$, as part of the same chromosome regions as the teleost GNAI1 genes (Figure 4). The phylogenetic analysis (Figure 2) is consistent with this, although the $V 1 A a$ and $V 1 A$ b sequences do not form two well-supported clusters. In the teleosts we also identified local duplicates of $O T R$, called OTR 1 and $O T R 2$, and of $V 2 A$, called $V 2 A 1$ and $V 2 A 2$.

\section{Evolution of the voltage-gated calcium channel, L-type gene family}

The CACNA1-L gene family is one of three subfamilies of voltage-gated calcium channel alpha subunits. The CACNA1-L subfamily, which belongs to the paralogon described herein, has four members in mammals and up to seven members in teleost fish. A more comprehensive analysis of this gene region and the remaining two voltage-gated calcium channel families, located in other paralogons, is in progress (Widmark et al.).

The phylogenetic analyses (Additional file 2: Figures S7 and S8) as well as the analyses of conserved synteny performed in this study show that the CACNA1-L gene family expanded in $2 \mathrm{R}$, before the radiation of vertebrates, with one ancestral gene giving rise to the $C A C$ NA1S, CACNA1D, CACNA1C and CACNA1F subtype genes. Subsequently the CACNA1S, $-1 D$ and $-1 F$ genes duplicated in $3 \mathrm{R}$ as part of the visual opsin gene-housing chromosome regions (Figure 5). As for several other gene families in this paralogon, these teleost duplicates have been translocated between the paralogous chromosome regions. For instance, teleost CACNA1S, CACNA1D and $C A C N A 1 F$ genes have all been translocated to zebrafish chromosome 8 (Figure 4) and stickleback linkage group XII (Additional file 5). As mentioned previously, we have observed similar rearrangements for other regions in teleost genomes during analyses of the somatostatin receptor gene regions [53]. The CACNA1D teleost cluster is not well resolved in the phylogenetic maximum likelihood analysis (Additional file 2: Figure S8), and no putative spotted gar sequence could be identified in the orthology predictions. However, our conserved synteny analyses as well as previous whole-genome analyses [10,45] are consistent with our conclusions.

\section{Implications for early vertebrate radiation}

Our analyses show that the gnathostome opsin repertoire with the four subtypes $R H 1, R H 2, S W S 1, S W S 2$ clearly resulted from a quadruplication of an ancestral chromosome block (that also included the adjacent but more distantly related $L W S$ genes without surviving duplicates). The presence of orthologs of all these 
genes in the pouched lamprey (Geotria australis) [7] imply that these chromosome-based gene duplications took place before the lamprey-gnathostome divergence, as previously proposed [54]. Our present extensive characterization of these paralogous gene regions in a broad range of vertebrates demonstrates that they resulted from the quadruplication of a very large chromosomal block in the time window of $2 \mathrm{R}$. Thus, it would follow that the lamprey lineage diverged after the two basal vertebrate tetraploidizations. If hagfishes and lampreys together form a monophyletic clade in the superclass Cyclostomata, as seems to be the case [55], this would mean that all vertebrates, including hagfish [56,57], share a common ancestor that had gone through $2 \mathrm{R}$. To our knowledge, the opsin gene family is presently the only one that has been found to have retained a complete $2 \mathrm{R}$ quartet in a lamprey. Several previously investigated gene families in the literature display fewer family members in lampreys than in gnathostomes see for instance $[20,58]$, suggesting more extensive gene loss in the lamprey lineage after 2R. Nevertheless, some incomplete gene families are consistent with post-2R divergence of cyclostomes and jawed vertebrates, including the retinoic acid receptors RAR [54]. The genomewide duplication pattern in the recently published sea lamprey (Petromyzon marinus) whole-genome sequence was found to be indicative of $2 \mathrm{R}$ [59].

\section{Conclusions}

We present an extensive analysis of the paralogous chromosome regions housing the gene families for visual opsins, the G-protein alpha subunit families for transducin (GNAT) and adenylyl cyclase inhibition (GNAI), the oxytocin and vasopressin receptors (OT/VP-R), and the L-type voltage-gated calcium channel alpha subunits (CACNA1-L), using both phylogenetic analyses and positional data for these as well as 34 chromosomal neighboring gene families. This combined dataset makes it possible to make a robust inference how this region evolved. We conclude that these related chromosome regions originated from an ancestral chromosome that was duplicated in the two basal vertebrate tetraploidizations (2R) resulting in four paralogous chromosome regions. The paralogon was duplicated again in the teleost-specific tetraploidization (3R) giving teleost fish additional gene family members. We could detect extensive post-3R chromosomal rearrangements between the paralogous chromosome regions in teleost genomes that obscure the view of these whole genome duplications, as noted previously $[45,46,53]$. However, the analyses of extant teleost genomes combined with the data presented here from the spotted gar, an out-group to teleost evolution, allowed us to resolve the rearrangements.
Referring also to our previous analyses of several gene families, we conclude that the basal vertebrate tetraploidizations contributed with new genetic material in several gene families involved in the phototransduction cascade, but also in other functions related to the vertebrate nervous system.

\section{Methods}

\section{Sequence identification and genome database searches}

Amino acid sequence predictions of the visual opsin genes were retrieved from the Ensembl genome browser (http://www.ensembl.org) [60] from a representative selection of vertebrate clades: human (Homo sapiens), mouse (Mus musculus), grey short-tailed opossum (Monodelphis domestica), anole lizard (Anolis carolinensis), chicken (Gallus gallus), Western clawed frog (Xenopus (Silurana) tropicalis), coelacanth (Latimeria chalumnae), zebrafish (Danio rerio), three-spined stickleback (Gasterosteus aculeatus), medaka (Oryzias latipes) and spotted green pufferfish (Tetraodon nigroviridis). Amino acid sequence predictions from the spotted gar (Lepisosteus oculatus) genome were identified through BLAST searches against the LepOcul assembly available at the Pre! Ensembl genome browser (http://pre.ensembl.org/ Lepisosteus_oculatus/Info/Index) and included in the analyses. Chicken data was complemented with cloned sequences (GenBank accession numbers: NP_990769.1, P22329.1 and P28682.1) [6,61]. Additionally, published visual opsin amino acid sequences from the pouched lamprey (Geotria australis) were used (GenBank accession numbers AAR14682.1, AAR14683.1, AAR14681.1, AAR14684.1 and AAR14680.1) [36].

For the oxytocin and vasopressin receptor gene family the same species repertoire as the visual opsins was used with a few exceptions: the Japanese pufferfish (Takifugu rubripes) was used instead of the related spotted green pufferfish and Southern platyfish (Xiphophorus maculatus) sequences (retrieved from Ensembl version 68) were added to increase resolution of the teleost branches. In addition, published oxytocin and vasopressin receptor sequences from the elephant shark (Callorhinchus milii) (GenBank accession numbers AB665985.1, AB665982.1, AB665983.1, AB665984.1, and AB671271.1) were added [29]. A sequence from the tunicate transparent sea squirt (Ciona intestinalis) was also added. Known OT/VP-R gene family members in the common octopus (Octopus vulgaris) were used as out-group, as described in [30].

For the CACNA1-L gene family the same repertoire as the visual opsins were used excluding Western clawed frog, coelacanth and spotted gar. Invertebrate sequences from transparent sea squirt and fruit fly (Drosophila melanogaster) were included for relative dating and as a root, respectively. 
Sequences were identified in several Ensembl versions for the different gene families, using Ensembl's automatic protein family predictions, spanning between Ensembl 60 (November 2010) to Ensembl 68 (July 2012). For the visual opsin gene family, gene predictions were identified through Ensembl's gene tree function [62] searches since the $O P N$ genes are spread among several Ensembl protein family predictions. All sequences and database identifiers have been verified against the genome assembly versions in Ensemble database version 71 (April 2013). This information can be found in Additional files 1 and 3. To identify additional family members that may have been excluded from the automatic Ensembl protein family predictions, Basic Local Alignment Searches (BLAST) [63] were performed using identified amino acid sequences (tblastn) as search terms with standard settings on the Ensembl database and the National Center for Biotechnology Information (NCBI) databases.

\section{Conserved synteny analysis}

The locations of the identified visual opsin, OT/VP-R, GNAT, GNAI and CACNA1-L sequences were recorded for the genome assemblies detailed above. All locations were verified against the genome assembly versions in Ensemble database version 71 (April 2013). Using the BioMart function, lists of gene predictions corresponding to the chromosome blocks bearing these genes in the human genome assembly GRCh 37 were downloaded from the Ensembl genome browser (versions specified below). This dataset was complemented with lists from the zebrafish genome (assembly Zv9) in some cases (see below). Chromosome blocks were defined as approximately $5 \mathrm{Mb}$ in each direction of each of the genes in the above-mentioned gene families.

From these lists of syntenic gene predictions we identified Ensembl protein family predictions that had members on at least two of the included chromosome blocks: these protein family predictions represent the syntenic or neighboring gene families. Amino acid sequence predictions corresponding to each of the identified neighboring gene families were downloaded from the Ensembl genome browser in order to do amino acid sequence alignments and phylogenetic analyses, and the locations of all identified sequences were recorded. The species included in these analyses were: human, mouse, grey short-tailed opossum, chicken, three-spined stickleback, spotted green pufferfish, medaka, zebrafish, transparent sea squirt (Ciona intestinalis or Ciona savignyi) and fruit fly (Drosophila melanogaster). Sequence predictions from the following additional species were used in some families in order to ensure phylogenetic signal and/or taxonomic representation: Tasmanian devil (Sarcophilus harrisii), zebra finch (Taeniopygia guttata), anole lizard, Western clawed frog, Japanese pufferfish
(Takifugu rubripes), Florida lancelet (Branchiostoma floridae) and the nematode Caenorhabditis elegans. Additional members that were not included in the automatic protein family predictions were identified by tblastn searches as described above. All sequences, database identifiers and locations have been verified against the genome assembly versions in Ensemble database version 71 (April 2013). For some of the neighboring gene families invertebrate sequences had to be identified by Hidden Markov Model searches (HMMER) using the HMMER web server (http:// hmmer.janelia.org/) [64] and its pHMMER implementation against the UniProtKB database and the NCBI non-redundant (NR) protein database.

The identification of neighboring gene families was done independently for the regions corresponding to each of the visual opsin, OT/VP-R, GNAT/GNAI and CACNA1-L gene families. The neighboring gene families were subsequently pooled together into one dataset and used for the analyses of conserved synteny. Since several of the chromosome blocks used in the analyses overlapped, some of the gene families were identified twice.

\section{Selection of neighboring gene families in the visual opsin chromosome regions}

The chromosome regions bearing the visual opsin genes OPN1SW, RHO, OPN1LW and OPN1MW in the human genome were used to select neighboring gene families. The chromosome blocks range between map positions $124 \mathrm{Mb}$ and $134 \mathrm{Mb}$ on chromosome 3, between map positions $123 \mathrm{Mb}$ and $133 \mathrm{Mb}$ on chromosome 7, and between map positions $148 \mathrm{Mb}$ and $158 \mathrm{Mb}$ on the $\mathrm{X}$ chromosome in Ensembl 60 (November 2010). Ensembl protein families with members present in at least two of the above-mentioned chromosomal regions were included in the subsequent phylogenetic analyses.

\section{Selection of neighboring gene families in the OT/VP-R chromosome regions}

Since not all identified OT/VP-R genes could be identified in the human genome (Ocampo Daza et al 2012), both the zebrafish and the human genomes were used to identify neighboring gene families for the analysis of conserved synteny. Both sets of chromosome blocks were downloaded from Ensembl 60 (November 2010).

The chromosome blocks in the zebrafish genome range between map positions $3 \mathrm{Mb}$ and $12.5 \mathrm{Mb}$ as well as between map positions $12.5 \mathrm{Mb}$ and $22 \mathrm{Mb}$ on chromosome 4, between map positions $37 \mathrm{Mb}$ and $52 \mathrm{Mb}$ on chromosome 6 , between map positions $13 \mathrm{Mb}$ and $30 \mathrm{Mb}$ on chromosome 23 and between map positions $1 \mathrm{bp}$ and $7 \mathrm{Mb}$ on chromosome 25. Since there are several OT/VP$\mathrm{R}$ genes located on the same chromosomes, these blocks do not necessarily represent $5 \mathrm{Mb}$. For example, the V1aR1-type and V2bR2-type genes are both located on 
chromosome 4, but to investigate the paralogy relationship between these two genes the chromosome blocks were treated separately. Ensembl protein families with members on at least three of the chromosome blocks were selected for the analysis of conserved synteny.

In the human genome the chromosome blocks range between map positions $199 \mathrm{Mb}$ and $209 \mathrm{Mb}$ on chromosome 1 , between map positions $3 \mathrm{Mb}$ and $13 \mathrm{Mb}$ on chromosome 3, between Map positions $56 \mathrm{Mb}$ and $66 \mathrm{Mb}$ on chromosome 12, and between map positions $147 \mathrm{Mb}$ and $157 \mathrm{Mb}$ on the $\mathrm{X}$ chromosome. Protein families with members on at least two of the chromosome blocks were selected.

\section{Selection of neighboring gene families in the CACNA1-L chromosome regions}

A list of all gene predictions located in the chromosome blocks between map positions $196 \mathrm{Mb}$ and $206 \mathrm{Mb}$ on chromosome 1, 48.5 $\mathrm{Mb}$ and $58.5 \mathrm{Mb}$ on chromosome 3, $1 \mathrm{bp}$ and $7 \mathrm{Mb}$ on chromosome 12 and between map positions $44 \mathrm{Mb}$ and $54 \mathrm{Mb}$ on chromosome $\mathrm{X}$ in the human genome were downloaded from Ensembl 61 (February 2011). These blocks represent the chromosomal regions of the CACNA1S, CACNA1D, CACNA1C and $C A C N A 1 F$ genes respectively. Ensembl protein families with members located on at least two of the chromosome blocks were considered for the analysis of conserved synteny.

\section{Selection of neighboring gene families in the GNAT and GNAI chromosome regions}

Lists of genes in the GNAT-GNAI bearing chromosome regions were downloaded from Ensembl 59 (Aug 2010). The chromosome blocks range between map positions 74.8 $\mathrm{Mb}$ and $84.7 \mathrm{Mb}$ on chromosome 7, between map positions $45.3 \mathrm{Mb}$ and $55.2 \mathrm{Mb}$ on chromosome 3, and between map positions $106 \mathrm{Mb}$ and 115.1 $\mathrm{Mb}$ on chromosome 1 in the human genome. Ensembl protein families represented on at least two of the three human chromosome blocks were included in the analysis of conserved synteny.

\section{Sequence annotation and curation}

For short, incomplete or highly diverging sequences among the identified gene predictions, the genomic sequences, including intronic and flanking sequences, were collected and the GenScan gene prediction server (http://genes.mit. edu/GENSCAN.html) [65] was used to ratify faulty exon predictions or to predict exons or entire genes de novo. Whenever possible, short Ensembl predictions were replaced with NCBI RefSeq sequences identified by BLAST searches, or with overlapping GenScan-predictions included in the Ensembl browser. Sequences that were still divergent with regard to exon-intron boundaries were curated manually by following consensus for splice donor and acceptor sites as well as sequence homology to other family members. Remaining highly divergent and unalignable sequence stretches in some of these predictions were removed. Short amino acid sequences that could not be ratified and did not provide enough sequence information in the alignments were removed entirely in order to prevent artifacts in the phylogenetic analyses. However the chromosomal locations of the gene predictions were registered.

\section{Sequence alignment and phylogenetic analyses}

Amino acid sequences were aligned using the ClustalW algorithm (Gonnet weight matrix, gap opening penalty 10.0 and gap extension penalty 0.20) [66] or the MUSCLE algorithm (with 16 iterations) [67] and the resulting alignments were inspected manually in order to ratify faulty or divergent predictions and curate misaligned sequence stretches. The manually curated alignments were used to calculate phylogenetic trees, using both the Neighbor Joining (NJ) and Phylogenetic Maximum Likelihood (PhyML) methods.

$\mathrm{NJ}$ trees with non-parametric bootstrap support were made using standard settings (NJ clustering algorithm with 1000 bootstrap iterations) in ClustalX 2.0.12 [66]. PhyML trees were made using the PhyML3.0 algorithm [68] with the following settings: amino acid frequencies (equilibrium frequencies), proportion of invariable sites (with optimized p-invar) and gamma shape parameters were estimated from the alignments, the number of substitution rate categories was set to 8 , BIONJ was chosen to create the starting tree, both NNI and SPR tree optimization methods were considered and both tree topology and branch length optimization were chosen. The amino acid substitution model was selected for each alignment using ProtTest3.2 [69] with the following settings: Likelihood scores were computed selecting between the JTT, LG, DCMut, Dayhoff, WAG, Blosum62 and VT substitution model matrices with no add-ons and a Fixed BioNJ JTT-based starting tree. Based on these analyses the LG model was chosen for the visual opsin gene family and the JTT model for the OT/VP-R and CACNA1-L gene families. The JTT model was also chosen for the majority of the neighboring gene families, except for the B4GALNT, CACNA2D, COL, L1CAM, PLG, PPP, QSOX and UBA gene families where the WAG model was chosen, and the RPL and TWF gene families where the LG model was chosen. The visual opsin, OT/VP-R and CACNA1-L PhyML topologies are supported by nonparametric bootstrap tests with 100 iterations. The PhyML tree topologies for the neighboring families are supported by non-parametric $\mathrm{SH}$-like approximate likelihood ratio (aLRT) tests [68,70] since this method is faster. 


\section{Relative dating and outgroup choice}

For the majority of the phylogenetic analyses the identified fruit fly sequences were used as out-group to root the trees. In some cases where no fruit fly sequence could be identified, C. elegans or tunicate sequences were used instead. The inclusion of lancelet or tunicate sequences in the phylogenetic analyses provides the relative dating for the time-window of $2 \mathrm{R}$. For a few families where no invertebrate sequences could be identified, midpoint-rooting [71] was used. The rooting of the phylogenetic trees for the neighboring gene families is summarized in Table 2 . The OT/VP-R gene family was rooted with identified Octopus vulgaris family members, as described in [30]. The visual opsin gene family was rooted with the human OPN3 gene.

\section{Analysis of conserved synteny between the human and spotted gar genomes}

A FASTA file containing all preliminary protein sequence predictions from the spotted gar genome assembly (LepOcu1) available in the Pre! Ensembl database was downloaded using the FTP server (http://pre.ensembl. org/downloads.html, retrieved July 25, 2012). This file was used for orthology searches using InParanoid 4.1 [72,73] (with standard settings) against a FASTA file containing translations of all human canonical transcripts (available from the InParanoid website: http://inparanoid.sbc. su.se/download/7.0_current/sequences/processed/H.sapiens.fa. From the resulting global dataset of orthology matches between the spotted gar and human genomes, the best spotted gar matches (highest bootstrap support) for each human gene prediction used in this study were mined. The locations of each of these spotted gar protein prediction matches in the spotted gar genome assembly were recorded and charted.

\section{Description of additional files}

The data sets supporting the results of this article, including all sequence alignments and phylogenetic tree files, are available in figshare and have been cited in the article where appropriate - see references [35,42-44]. The following additional files are included with this article. Additional file 1 includes detailed information on the main gene families, such as database identifiers, location data and annotation notes for all analyzed sequences. Additional file 2 includes the phylogenetic trees of the main gene families (Figures S1-S8). Additional file 3 includes detailed information on the neighboring gene families. Additional file 4 includes supplementary notes on the phylogenies of neighboring gene families. Additional file 5 includes conserved synteny tables with the synteny data underlying our evolutionary scenario.

\section{Additional files}

Additional file 1: Tables including detailed information on the main gene families. Includes database identifiers, location data and annotation notes for all analyzed sequences.

Additional file 2: Figures S1-S8. Phylogenetic trees of the main gene families of visual opsins, oxytocin and vasopressin receptors (OTNP-R) and L-type voltage-gated calcium channels (CACNA1-L). Full lists of sequence names, locations and database identifiers for these families are included in Additional file 1.

Additional file 3: Tables including detailed information on the neighboring gene families. Includes database identifiers, location data and annotation notes for all analyzed sequences.

Additional file 4: Supplementary notes. Topologies of neighboring gene family trees.

Additional file 5: Tables of conserved synteny between the identified chromosome blocks in the human, chicken, spotted gar, zebrafish and three-spined stickleback genomes. Each table is included as a separate tab in the workbook.

\section{Competing interests}

The authors declare that they have no competing interests.

\section{Authors' contributions}

DLag, DOD, JW and GS participated in the study design and performed phylogenetic and chromosome analyses. XMA participated in the analyses and in the discussion of results. DLar conceived and co-designed the study and participated in the analyses. All authors co-wrote the article and have read and approved the final version.

\section{Acknowledgements}

This work was supported by grants from the Swedish Research Council and the Carl Trygger Foundation. We are grateful to Ola Gustafsson and Susanne Dreborg who participated in the initial stages of the opsin analyses, to Venkatesh Chandra Reddy who carried out initial phylogenetic analyses on some of the neighboring gene families, and to Christina A. Bergqvist and Lars G. Lundin for fruitful discussions throughout this project.

\section{Author details}

${ }^{1}$ Department of Neuroscience, Science for Life Laboratory, Uppsala University, Box 593, SE-75124 Uppsala, Sweden. ${ }^{2}$ Present address: Department of Medical Biochemistry and Microbiology, Science for Life Laboratory, Uppsala University, Box 582, SE-75123 Uppsala, Sweden.

Received: 27 June 2013 Accepted: 29 October 2013

Published: 2 November 2013

References

1. Hering L, Henze MJ, Kohler M, Kelber A, Bleidorn C, Leschke M, Nickel B, Meyer M, Kircher M, Sunnucks P, Mayer G: Opsins in onychophora (velvet worms) suggest a single origin and subsequent diversification of visual pigments in arthropods. Mol Biol Evol 2012, 29:3451-3458.

2. Davies WIL, Collin SP, Hunt DM: Molecular ecology and adaptation of visual photopigments in craniates. Mol Ecol 2012, 21:3121-3158.

3. Davies WL, Carvalho LS, Tay B-H, Brenner S, Hunt DM, Venkatesh B: Into the blue: gene duplication and loss underlie color vision adaptations in a deep-sea chimaera, the elephant shark Callorhinchus milii. Genome Res 2009, 19:415-426.

4. Collin SP: Evolution and ecology of retinal photoreception in early vertebrates. Brain Behav Evol 2010, 75:174-185.

5. Rennison DJ, Owens GL, Taylor JS: Opsin gene duplication and divergence in ray-finned fish. Mol Phylogenet Evol 2012, 62:986-1008.

6. Okano T, Kojima D, Fukada Y, Shichida Y, Yoshizawa T: Primary structures of chicken cone visual pigments: vertebrate rhodopsins have evolved out of cone visual pigments. Proc Natl Acad Sci USA 1992, 89:5932-5936.

7. Davies WL, Cowing JA, Carvalho LS, Potter IC, Trezise AEO, Hunt DM, Collin SP: Functional characterization, tuning, and regulation of visual pigment gene expression in an anadromous lamprey. FASEB J 2007, 21:2713-2724. 
8. Larhammar D, Lundin L-G, Hallböök F: The human Hox-bearing chromosome regions did arise by block or chromosome (or even genome) duplications. Genome Res 2002, 12:1910-1920.

9. Dehal $\mathrm{P}$, Boore $\mathrm{J} \mathrm{L}$ : Two rounds of whole genome duplication in the ancestral vertebrate. PLOS Biol 2005, 3:e314.

10. Nakatani $Y$, Takeda H, Kohara $Y$, Morishita S: Reconstruction of the vertebrate ancestral genome reveals dynamic genome reorganization in early vertebrates. Genome Res 2007, 17:1254-1265.

11. Putnam NH, Butts T, Ferrier DEK, Furlong RF, Hellsten U, Kawashima T, Robinson-Rechavi M, Shoguchi E, Terry A, Yu J-K, Benito-Gutiérrez E, Dubchak I, Garcia-Fernàndez J, Gibson-Brown JJ, Grigoriev IV, Horton AC, de Jong PJ, Jurka J, Kapitonov W, Kohara Y, Kuroki Y, Lindquist E, Lucas S, Osoegawa K, Pennacchio LA, Salamov AA, Satou Y, Sauka-Spengler T, Schmutz J, Shin-I T, et al: The amphioxus genome and the evolution of the chordate karyotype. Nature 2008, 453:1064-1071.

12. Jaillon O, Aury J-M, Brunet F, Petit J-L, Stange-Thomann N, Mauceli E, Bouneau L, Fischer C, Ozouf-Costaz C, Bernot A, Nicaud S, Jaffe D, Fisher S, Lutfalla G, Dossat C, Segurens B, Dasilva C, Salanoubat M, Levy M, Boudet N, Castellano S, Anthouard V, Jubin C, Castelli V, Katinka M, Vacherie B, Biémont C, Skalli Z Cattolico L, Poulain J, et al: Genome duplication in the teleost fish Tetraodon nigroviridis reveals the early vertebrate proto-karyotype. Nature 2004, 431:946-957.

13. Sundström G, Dreborg S, Larhammar D: Concomitant duplications of opioid peptide and receptor genes before the origin of jawed vertebrates. PLoS One 2010, 5:e10512.

14. Dreborg S, Sundström G, Larsson TA, Larhammar D: Evolution of vertebrate opioid receptors. Proc Natl Acad Sci USA 2008, 105:15487-15492.

15. Sundström G, Larsson TA, Brenner S, Venkatesh B, Larhammar D: Evolution of the neuropeptide $Y$ family: new genes by chromosome duplications in early vertebrates and in teleost fishes. Gen Comp Endocrinol 2008, 155:705-716.

16. Larsson TA, Tay B-H, Sundström G, Fredriksson R, Brenner S, Larhammar D, Venkatesh B: Neuropeptide Y-family peptides and receptors in the elephant shark, Callorhinchus milii confirm gene duplications before the gnathostome radiation. Genomics 2009, 93:254-260.

17. Larsson TA, Olsson F, Sundström G, Lundin L-G, Brenner S, Venkatesh B, Larhammar D: Early vertebrate chromosome duplications and the evolution of the neuropeptide Y receptor gene regions. BMC Evol Biol 2008, 8:184.

18. Widmark J, Sundström G: Ocampo Daza D, Larhammar D: Differential evolution of voltage-gated sodium channels in tetrapods and teleost fishes. Mol Biol Evol 2011, 28:859-871.

19. Ocampo Daza D, Sundström G, Bergqvist CA, Duan C, Larhammar D: Evolution of the insulin-like growth factor binding protein (IGFBP) family. Endocrinology 2011, 152:2278-2289.

20. Hultqvist G, Ocampo Daza D, Larhammar D, Kilimann MW: Evolution of the vertebrate paralemmin gene family: ancient origin of gene duplicates suggests distinct functions. PLOS One 2012, 7:e41850.

21. Lagman D, Sundström G, Ocampo Daza D, Abalo XM, Larhammar D: Expansion of Transducin Subunit Gene Families in Early Vertebrate Tetraploidizations. Genomics 2012:1-9.

22. Larhammar D, Nordström K, Larsson TA: Evolution of vertebrate rod and cone phototransduction genes. Phil Trans Roy Soc Lond B Biol Sci 2009, 364:2867-2880.

23. Nordström K, Larsson TA, Larhammar D: Extensive duplications of phototransduction genes in early vertebrate evolution correlate with block (chromosome) duplications. Genomics 2004, 83:852-872.

24. Kawamura S, Blow NS, Yokoyama S: Genetic analyses of visual pigments of the pigeon (Columba livia). Genetics 1999, 153:1839-1850.

25. Chinen A, Hamaoka T, Yamada Y, Kawamura S: Gene duplication and spectral diversification of cone visual pigments of zebrafish. Genetics 2003, 163:663-675.

26. Yokoyama R, Yokoyama S: Molecular characterization of a blue visual pigment gene in the fish Astyanax fasciatus. FEBS Lett 1993, 334:27-31.

27. Oka Y, Korsching SI: Shared and unique $G$ alpha proteins in the zebrafish versus mammalian senses of taste and smell. Chem Senses 2011, 36:357-365.

28. Ohmoto M, Okada S, Nakamura S, Abe K, Matsumoto I: Mutually exclusive expression of Gaia and Ga14 reveals diversification of taste receptor cells in zebrafish. J Comp Neurol 2011, 519:1616-1629.

29. Yamaguchi $Y$, Kaiya H, Konno N, Iwata E, Miyazato M, Uchiyama M, Bell JD, Toop T, Donald JA, Brenner S, Venkatesh B, Hyodo S: The fifth neurohypophysial hormone receptor is structurally related to the V2-type receptor but functionally similar to V1-type receptors. Gen Comp Endocrinol 2012, 178:519-528.

30. Ocampo Daza D, Lewicka M, Larhammar D: The oxytocin/vasopressin receptor family has at least five members in the gnathostome lineage, including two distinct V2 subtypes. Gen Comp Endocrinol 2012, 175:135-143.

31. Peirson SN, Halford S, Foster RG: The evolution of irradiance detection: melanopsin and the non-visual opsins. Phil Trans Roy Soc Lond B Biol Sci 2009, 364:2849-2865.

32. Davies WL, Hankins MW, Foster RG: Vertebrate ancient opsin and melanopsin: divergent irradiance detectors. Photochem Photobiol Sci 2010, 9:1444-1457.

33. Sato K, Yamashita T, Ohuchi H, Shichida Y: Vertebrate ancient-long opsin has molecular properties intermediate between those of vertebrate and invertebrate visual pigments. Biochemistry 2011, 50:10484-10490.

34. Kusakabe T, Tsuda M: Photoreceptive systems in ascidians. Photochem Photobiol 2007, 83:248-252.

35. Phylogenetic analyses of the visual opsin genes of the LWS, SWS1, SWS2, RHI and RH2 clades. http://dx.doi.org/10.6084/m9.figshare.705157.

36. Collin SP, Knight MA, Davies WL, Potter IC, Hunt DM, Trezise AEO: Ancient colour vision: multiple opsin genes in the ancestral vertebrates. Curr Biol 2003, 13:R864-R865.

37. Alföldi J, Di Palma F, Grabherr M, Williams C, Kong L, Mauceli E, Russell P, Lowe CB, Glor RE, Jaffe JD, Ray DA, Boissinot S, Shedlock AM, Botka C, Castoe TA, Colbourne JK, Fujita MK, Moreno RG, ten Hallers BF, Haussler D, Heger A, Heiman D, Janes DE, Johnson J, de Jong PJ, Koriabine MY, Lara M, Novick PA, Organ CL, Peach SE, et al: The genome of the green anole lizard and a comparative analysis with birds and mammals. Nature 2011 477:587-591.

38. Yokoyama S, Zhang H, Radlwimmer FB, Blow NS: Adaptive evolution of color vision of the Comoran coelacanth (Latimeria chalumnae). Proc Natl Acad Sci USA 1999, 96:6279-6284.

39. Mano H, Kojima D, Fukada Y: Exo-rhodopsin: a novel rhodopsin expressed in the zebrafish pineal gland. Brain research Molecular brain research 1999, 73:110-118.

40. Fitzgibbon J, Hope A, Slobodyanyuk SJ, Bellingham J, Bowmaker JK, Hunt DM: The rhodopsin-encoding gene of bony fish lacks introns. Gene 1995, 164:273-277.

41. Morrow JM, Lazic S, Chang BSW: A novel rhodopsin-like gene expressed in zebrafish retina. Vis Neurosci 2011, 28:325-335.

42. Phylogenetic analyses of the vertebrate oxytocin and vasopressin receptor gene family. http://dx.doi.org/10.6084/m9.figshare.707336

43. Phylogenetic analyses of the vertebrate voltage-gated calcium channel L-type alpha 1 subunit gene family. http://dx.doi.org/10.6084/m9.figshare.710637.

44. Phylogenetic analyses of syntenic gene families in visual opsin gene-bearing chromosome regions. http://dx.doi.org/10.6084/m9.figshare.705852.

45. Kasahara M, Naruse K, Sasaki S, Nakatani Y, Qu W, Ahsan B, Yamada T, Nagayasu Y, Doi K, Kasai Y, Jindo T, Kobayashi D, Shimada A, Toyoda A, Kuroki Y, Fujiyama A, Sasaki T, Shimizu A, Asakawa S, Shimizu N, Hashimoto S-I, Yang J, Lee Y, Matsushima K, Sugano S, Sakaizumi M, Narita T, Ohishi K, Haga S, Ohta F, et al: The medaka draft genome and insights into vertebrate genome evolution. Nature 2007, 447:714-719.

46. Amores A, Catchen J, Ferrara A, Fontenot Q, Postlethwait JH: Genome evolution and meiotic maps by massively parallel DNA sequencing: spotted gar, an outgroup for the teleost genome duplication. Genetics 2011, 188:799-808.

47. Bellingham J, Tarttelin EE, Foster RG, Wells DJ: Structure and evolution of the teleost extraretinal rod-like opsin (errlo) and ocular rod opsin (rho) genes is teleost rho a retrogene. J Exp Zool B Mol Dev Evol 2003, 297:1-10.

48. Venkatesh B, Ning Y, Brenner S: Late changes in spliceosomal introns define clades in vertebrate evolution. Proc Natl Acad Sci USA 1999 96:10267-10271.

49. Pointer MA, Carvalho LS, Cowing JA, Bowmaker JK, Hunt DM: The visual pigments of a deep-sea teleost, the pearl eye Scopelarchus analis. J Exp Biol 2007, 210(16):2829-2835.

50. Zhang H, Futami K, Horie N, Okamura A, Utoh T, Mikawa N, Yamada Y, Tanaka S, Okamoto N: Molecular cloning of fresh water and deep-sea rod opsin genes from Japanese eel Anguilla japonica and expressional analyses during sexual maturation. FEBS Lett 2000, 469:39-43.

51. Archer S, Hope A, Partridge JC: The molecular basis for the green-blue sensitivity shift in the rod visual pigments of the European eel. Proceedings Biological sciences/The Royal Society 1995, 262:289-295. 
52. Tan F, Lolait SJ, Brownstein MJ, Saito N, MacLeod V, Baeyens DA, Mayeux $\mathrm{PR}$, Jones SM, Cornett LE: Molecular cloning and functional characterization of a vasotocin receptor subtype that is expressed in the shell gland and brain of the domestic chicken. Biol Reprod 2000, 62:8-15.

53. Ocampo Daza D, Sundström G, Bergqvist CA, Larhammar D: The evolution of vertebrate somatostatin receptors and their gene regions involves extensive chromosomal rearrangements. BMC Evol Biol 2012, 12:231.

54. Kuraku S, Meyer A, Kuratani S: Timing of genome duplications relative to the origin of the vertebrates: did cyclostomes diverge before or after? Mol Biol Evol 2009, 26:47-59.

55. Heimberg AM, Cowper-Sal-lari R, Sémon M, Donoghue PCJ, Peterson KJ: microRNAs reveal the interrelationships of hagfish, lampreys, and gnathostomes and the nature of the ancestral vertebrate. Proc Natl Acad Sci U S A 2010, 107:19379-19383.

56. Ota KG, Fujimoto S, Oisi Y, Kuratani S: Identification of vertebra-like elements and their possible differentiation from sclerotomes in the hagfish. Nat Commun 2011, 2: . http://dx.doi.org/10.1038/ncomms1355.

57. Shimeld SM, Donoghue PCJ: Evolutionary crossroads in developmental biology: cyclostomes (lamprey and hagfish). Development 2012, 139:2091-2099.

58. Jakobsson J, Ackermann F, Andersson F, Larhammar D, Löw P, Brodin L: Regulation of synaptic vesicle budding and dynamin function by an EHD ATPase. J Neurosci 2011, 31:13972-13980.

59. Smith JJ, Kuraku S, Holt C, Sauka-Spengler T, Jiang N, Campbell MS, Yandell MD, Manousaki T, Meyer A, Bloom OE, Morgan JR, Buxbaum JD, Sachidanandam R, Sims C, Garruss AS, Cook M, Krumlauf R, Wiedemann LM, Sower SA, Decatur WA Hall JA, Amemiya CT, Saha NR, Buckley KM, Rast JP, Das S, Hirano M, McCurley N, Guo P, Rohner N, et al: Sequencing of the sea lamprey (Petromyzon marinus) genome provides insights into vertebrate evolution. Nat Genet 2013, 45:415-421.

60. Flicek P, Amode MR, Barrell D, Beal K, Brent S, Carvalho-Silva D, Clapham P, Coates G, Fairley S, Fitzgerald S, Gil L, Gordon L, Hendrix M, Hourlier T, Johnson N, Kähäri AK, Keefe D, Keenan S, Kinsella R, Komorowska M, Koscielny G, Kulesha E, Larsson P, Longden I, McLaren W, Muffato M, Overduin B, Pignatelli M, Pritchard B, Riat HS, et al: Ensembl 2012. Nucleic Acids Res 2012, 40:D84-D90.

61. Tokunaga F, Iwasa T, Miyagishi M, Kayada S: Cloning of cDNA and amino acid sequence of one of chicken cone visual pigments. Biochem Biophys Res Commun 1990, 173:1212-1217.

62. Vilella AJ, Severin J, Ureta-Vidal A, Heng L, Durbin R, Birney E: EnsemblCompara GeneTrees: Complete, duplication-aware phylogenetic trees in vertebrates. Genome Res 2009, 19:327-335.

63. Altschul SF, Gish W, Miller W, Myers EW, Lipman DJ: Basic local alignment search tool. J Mol Biol 1990, 215:403-410.

64. Finn RD, Clements J, Eddy SR: HMMER web server: interactive sequence similarity searching. Nucleic Acids Res 2011, 39(2):W29-W37.

65. Burge C, Karlin S: Prediction of complete gene structures in human genomic DNA. J Mol Biol 1997, 268:78-94.

66. Larkin MA, Blackshields G, Brown NP, Chenna R, McGettigan PA, McWilliam H, Valentin F, Wallace IM, Wilm A, Lopez R, Thompson JD, Gibson TJ, Higgins DG: Clustal W and Clustal X version 2.0. Bioinformatics 2007, 23:2947-2948.

67. Edgar RC: MUSCLE: multiple sequence alignment with high accuracy and high throughput. Nucleic Acids Res 2004, 32:1792-1797.

68. Guindon S, Dufayard J-F, Lefort V, Anisimova M, Hordijk W, Gascuel O: New algorithms and methods to estimate maximum-likelihood phylogenies: assessing the performance of PhyML 3.0. Syst Biol 2010, 59:307-321.

69. Abascal F, Zardoya R, Posada D: ProtTest: selection of best-fit models of protein evolution. Bioinformatics 2005, 21:2104-2105.

70. Anisimova M, Gascuel O: Approximate likelihood-ratio test for branches: A fast, accurate, and powerful alternative. Syst Biol 2006, 55:539-552.
71. Hess PN, De Moraes Russo CA: An empirical test of the midpoint rooting method. Biol J Linn Soc 2007, 92:669-674.

72. Berglund A-C, Sjölund E, Ostlund G, Sonnhammer ELL: InParanoid 6: eukaryotic ortholog clusters with inparalogs. Nucleic Acids Res 2008, 36:D263-D266.

73. Remm M, Storm CE, Sonnhammer EL: Automatic clustering of orthologs and in-paralogs from pairwise species comparisons. J Mol Biol 2001, 314:1041-1052.

doi:10.1186/1471-2148-13-238

Cite this article as: Lagman et al:: The vertebrate ancestral repertoire of visual opsins, transducin alpha subunits and oxytocin/vasopressin receptors was established by duplication of their shared genomic region in the two rounds of early vertebrate genome duplications. $B M C$ Evolutionary Biology 2013 13:238.

\section{Submit your next manuscript to BioMed Central and take full advantage of:}

- Convenient online submission

- Thorough peer review

- No space constraints or color figure charges

- Immediate publication on acceptance

- Inclusion in PubMed, CAS, Scopus and Google Scholar

- Research which is freely available for redistribution

Submit your manuscript at www.biomedcentral.com/submit
C BioMed Central 